\title{
Epigenetic silencing of microRNA-149 in cancer-associated fibroblasts mediates prostaglandin E2/interleukin-6 signaling in the tumor microenvironment
}

$\mathrm{Pu} \mathrm{Li}^{1, *}$, Jing-Xuan Shan ${ }^{3, *}$, Xue-Hua Chen ${ }^{1,{ }^{*}}$, Di Zhang ${ }^{2, *}$, Li-Ping Su ${ }^{1}$, Xiu-Ying Huang ${ }^{2}$, Bei-Qin Yu ${ }^{1}$, Qiao-Ming Zhi ${ }^{1}$, Cheng-Long $\mathrm{Li}^{1}$, Ya-Qing Wang ${ }^{2}$, Sara Tomei ${ }^{3}$, Qu Cai ${ }^{1}$, Jun $\mathrm{Ji}^{1}$, Jian-Fang $\mathrm{Li}^{1}$, Lotfi Chouchane ${ }^{3}$, Ying-Yan Yu ${ }^{1}$, Fang-Zhen Sun ${ }^{2}$, Zhi-Heng Xu ${ }^{2}$, Bing-Ya Liu ${ }^{1}$, Zheng-Gang Zhu ${ }^{1}$

${ }^{I}$ Department of Surgery, Shanghai Key Laboratory of Gastric Neoplasms, Shanghai Institute of Digestive Surgery, Ruijin Hospital, School of Medicine, Shanghai Jiao Tong University, 197 Ruijin Er Rd, Shanghai 200025, China; ${ }^{2}$ State Key Laboratory of Molecular Developmental Biology, Institute of Genetics and Developmental Biology, Chinese Academy of Sciences, 1 West Beichen Rd, Beijing 100101, China; ${ }^{3}$ Laboratory of Genetic Medicine and Immnology, Weill Cornell Medical College in Qatar, Education City, P.O.Box 24144, Doha, Qatar

Tumor initiation and growth depend on its microenvironment in which cancer-associated fibroblasts (CAFs) in tumor stroma play an important role. Prostaglandin E2 (PGE2) and interleukin (IL)-6 signal pathways are involved in the crosstalk between tumor and stromal cells. However, how PGE2-mediated signaling modulates this crosstalk remains unclear. Here, we show that microRNA (miR)-149 links PGE2 and IL-6 signaling in mediating the crosstalk between tumor cells and CAFs in gastric cancer (GC). miR-149 inhibited fibroblast activation by targeting IL-6 and miR-149 expression was substantially suppressed in the CAFs of GC. miR-149 negatively regulated CAFs and their effect on GC development both in vitro and in vivo. CAFs enhanced epithelial-to-mesenchymal transition (EMT) and the stem-like properties of GC cells in a miR-149-IL-6-dependent manner. In addition to IL-6, PGE2 receptor 2 (PTGER2/EP2) was revealed as another potential target of miR-149 in fibroblasts. Furthermore, $H$. pylori infection, a leading cause of human GC, was able to induce cyclooxygenase-2 (COX-2)/PGE2 signaling and to enhance PGE2 production, resulting in the hypermethylation of miR-149 in CAFs and increased IL-6 secretion. Our findings indicate that miR-149 mediates the crosstalk between tumor cells and CAFs in GC and highlight the potential of interfering miRNAs in stromal cells to improve cancer therapy.

Keywords: microRNA-149; epigenetic silencing; cancer-associated fibroblasts; PGE2; IL-6

Cell Research (2015) 25:588-603. doi:10.1038/cr.2015.51; published online 28 April 2015

\section{Introduction}

The tumor stroma, including the GC stroma, consists of the extracellular matrix and various cells including

\footnotetext{
*These four authors contributed equally to this work. Correspondence: Zheng-Gang Zhu ${ }^{\mathrm{a}}$, Bing-Ya Liu ${ }^{\mathrm{b}}$, Zhi-Heng Xu ${ }^{\mathrm{c}}$

${ }^{a}$ Tel: 86-21-6433-3414; Fax: 86-21-6433-3414

E-mail: zhenggang.zhu@yahoo.com

bE-mail: byliu@sjtu.edu.cn

${ }^{\mathrm{c} T e l}$ : 86-10-6484-0829

E-mail: zhxu@genetics.ac.cn

Received 2 July 2014; revised 27 November 2014; accepted 17 December 2014; published online 28 April 2015
}

fibroblasts and inflammatory cells. Among them, cancer-associated fibroblasts (CAFs), play a critical role in promoting tumor growth, invasion and angiogenesis [1]. CAFs are usually considered to be derived from normal fibroblasts (NFs) that are in close contact with tumor cells [2]. Elevated expression of pro-inflammatory genes is essential to maintain the characteristics of CAFs [3]. On the other hand, neoplastic cells can induce the transformation of fibroblast cells to adapt to the tumor microenvironment and co-evolve with tumor cells to foster malignancy [2].

CAFs and neoplastic cells communicate via growth factors and inflammation factors [4]. Inflammatory signaling pathways are emerging as highly promising 
targets to block the communication between tumor cells and CAFs [5]. Among them, cyclooxygenase-2 (COX2)/prostaglandin E2 (PGE2) signaling, which was discovered in transformed epithelial cells [6], contributes uniquely to tumorigenesis through increasing the population of tumor initiating cells (TICs) [7]. Overexpression of COX-2 is sufficient to induce tumor formation in transgenic mouse models [8], and has been proposed to exert its pro-tumor effects through the production of PGE2 [9]. PGE2 promotes tumor development by stimulating angiogenesis, inhibiting apoptosis, increasing invasion, and modulating inflammatory and immune responses [10]. In addition to tumor cells, elevated stromal PGE2 levels could be ascribed to fibroblasts $[11,12]$. Although the role of COX-2/PGE2 signaling in tumorigenesis has been studied extensively, the mechanism underlying the communication between tumor cells and fibroblasts remains unclear.

Increased PGE2 levels induce the expression of the inflammatory factor interleukin (IL)-6 in fibroblasts [13, 14]. IL-6 is an important mediator involved in the crosstalk between CAFs and tumor cells $[3,15]$. Contrary to PGE2, CAF-producing IL-6 is likely to play a role mainly in the cancer cell niche $[3,15-18]$. The presence of tumor cells enhances the release of IL- 6 by CAFs [16]. Recent findings indicate that IL-6 can also increase PGE2 production $[19,20]$, suggesting that IL-6 signaling is essential for the activation of the COX-2/PGE2 pathway in cancer cells [16]. Therefore, a positive PGE2-IL-6 feedback loop is likely to be involved in the crosstalk between the tumor and its stroma.

Dysregulation of microRNAs (miRNAs) is associated with tumorigenesis [21] and fibroblast reprogramming in cancer [22]. Most studies, including those from our group, have focused on miRNAs in tumor cells, their aberrant expression and their target genes [23, 24], whereas miRNAs that do not change in tumor tissues are usually ignored. Recent reports suggest that miRNAs are aberrantly expressed in CAFs compared to NFs in endometrial, bladder, breast and ovarian cancers [22, 25-27]. The deregulation of miRNAs contributes to the pro-tumor ability of CAF through altering the expression of their target genes. However, tumor tissues consist of different cell types, of which CAFs occupy only a small percentage. Deregulation of gene or protein in these cells is usually missed in whole-tissue analysis. Genetic mutations in CAFs are rare, implying that an alternative way of gene regulation may be active [28, 29]. A genome-wide analysis of stromal cells from breast cancer suggests that epigenetic changes are likely to be the main regulatory mechanism in CAFs [30]. Therefore, it is possible that altered miRNA expression in CAFs may fine-tune PGE2 signaling to orchestrate the interaction between CAFs and neoplastic cells.

In the present study, we identify miRNAs that are deregulated in CAFs and investigate the mechanisms underlying their dysregulation as well as their role in tumorigenesis.

\section{Results}

miR-149 targets IL-6 mRNA and is downregulated in GC CAFs

To identify miRNAs that may play a role in tumorigenesis, the miR profiles of 28 pairs of gastric tumors and para-tumors were analyzed by microarray (Supplementary information, Figure S1A). The roles of several significantly deregulated miRNAs in gastric tumors have been described [20, 21]. Here, we focused on miRNAs whose expression did not differ between tumor and para-tumor tissues. CAFs play an important role in tumorigenesis, and miRNAs can reprogram NFs into CAFs [22]. The conversion of NFs to CAFs occurs through a mechanism similar to that of fibrosis [31], in which miRNAs, such as miR-21 and miR-29, are indispensable $[32,33]$. To identify CAF-specific deregulated miRNAs, we searched published datasets for miRNAs deregulated in CAFs in breast cancer [27] and cardiomyocyte fibrosis [34], and compared them with miRNAs unchanged in our array data (Supplementary information, Figure S1B). The three groups shared one common miR, miR149 , which is dramatically downregulated in fibrosis and CAFs.

A search of target predicting tools (TargetScan, Microrna.org, PicTar and RNAhybrid) [35] for potential target genes of miR-149 identified $I L-6$, which is CAF specific and associated with tumorigenesis [16-18] (Figure 1A). To validate the predicted role of IL-6 as a target gene of miR-149, wild-type and mutant 3'-UTR fragments of IL- 6 were cloned into the pMIR-REPORT luciferase vector (Supplementary information, Figure S2). The constructs were co-transfected with miR-149 mimics or control into fibroblasts followed by luciferase reporter assay. The relative luciferase activity of pMIR/ $I L-6-3^{\prime}-\mathrm{UTR}^{\mathrm{wt}}$, but not that of pMIR/IL-6-3'-UTR ${ }^{\mathrm{mut}}$, was significantly suppressed by miR-149 mimics. By contrast, anti-miR-149 enhanced the relative luciferase activity of pMIR/IL-6-3'-UTR ${ }^{\mathrm{wt}}$, but not that of $\mathrm{pMIR} / I L-6-3^{\prime}-$ $\mathrm{UTR}^{\text {mut }}$ (Figure 1B).

Based on the results indicating that miR-149 targets IL-6 mRNA, we investigated the relationship between miR-149 and IL-6 and their role in GC. To confirm the downregulation of miR-149 in CAFs, five primary CAF and NF cell lines were established from gastric malignant 
A

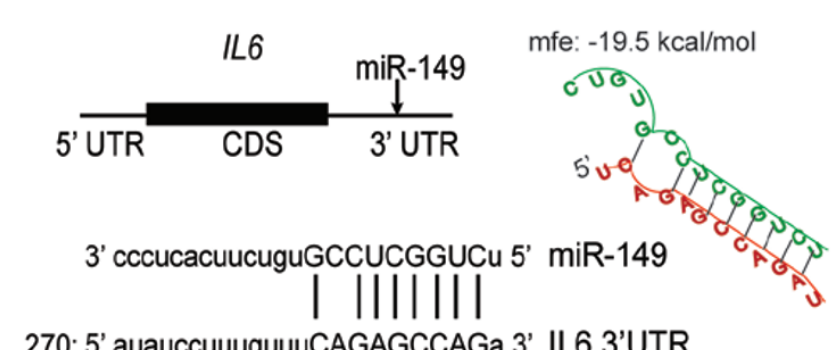

270: 5' auauccuuuguuuCAGAGCCAGa 3' IL6 3'UTR

C

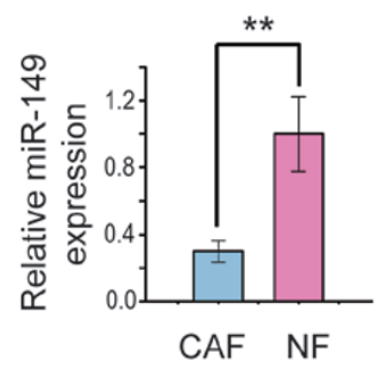

D

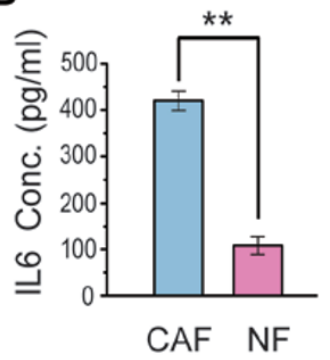

B

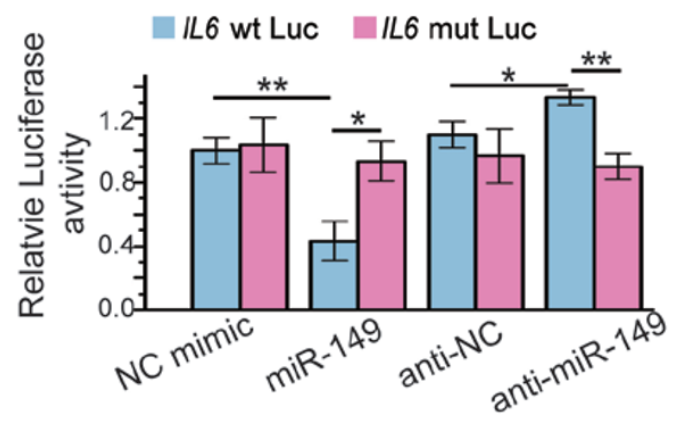

E

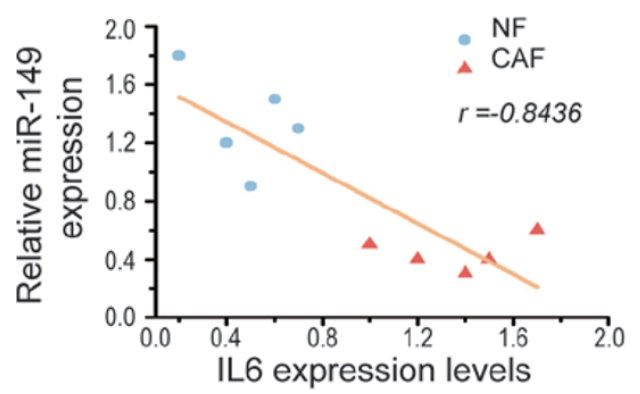

F

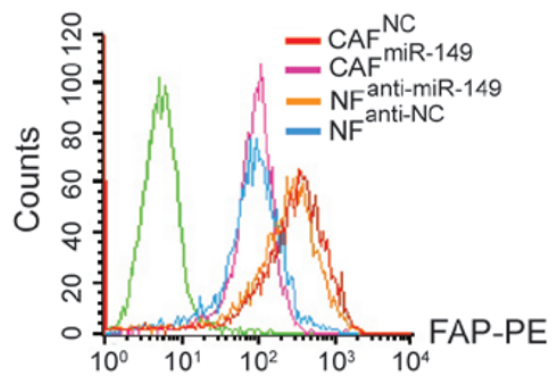

I

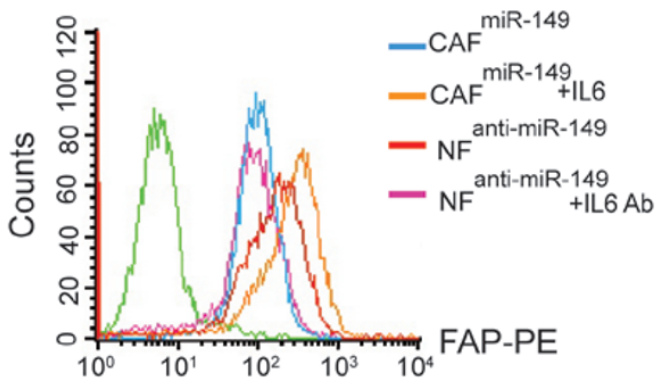

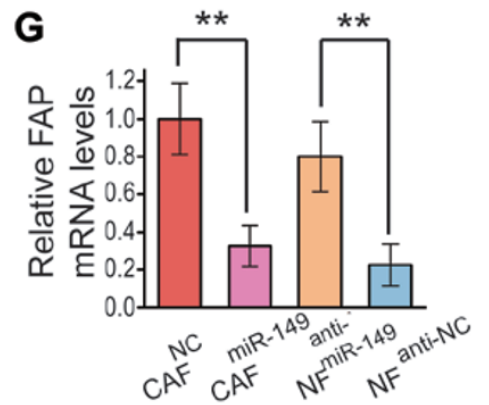

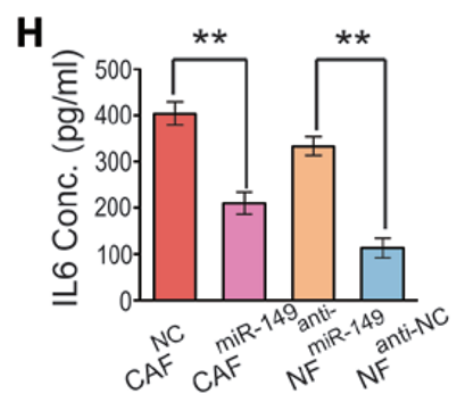

J

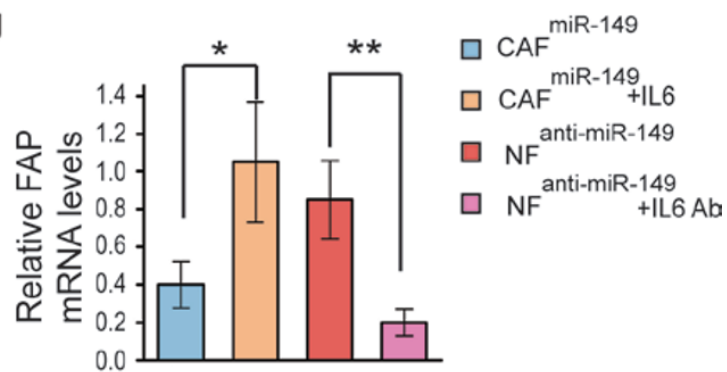

Figure 1 miR-149 targets IL-6 in gastric stroma fibroblasts and regulates FAP expression via IL-6. (A) Schematic graph of 3'-UTR of IL-6 with the putative binding sites of miR-149. The minimum free energy (mfe) required for RNA hybridization was predicted by RNAhybrid software (mfe: $-19.5 \mathrm{kcal} / \mathrm{mol})$. (B) Effect of miR-149 mimics and miR-149 inhibitor on IL-6 expression. Luciferase activity in fibroblasts co-transfected with reporter vector with wild-type or mutant IL-6 3'-UTR and miR149 mimics or inhibitors. (C) The miR-149 expression levels in 5 CAFs and 5 NFs established from gastric tumor tissues and matched para-tumor tissues were quantified by qRT-PCR. (D) Concentrations of IL-6 in the media of cultured CAF and NF cell lines were analyzed by ELISA. (E) The levels of miR-149 expression correlate inversely with IL-6 expression in CAFs and NFs. (F) The FAP expression levels in CAF or NF transfected with controls, miR-149 or anti-miR-149 (CAF ${ }^{\mathrm{NC}}, \mathrm{CAF}^{\mathrm{miR}-149}$, $N^{\text {anti-miR-149 }}$ and NF ${ }^{\text {anti-NC}}$, respectively) as analyzed by flow cytometry. (G) The relative FAP mRNA levels in CAF ${ }^{N C}, C^{\prime} F^{\text {miR-149' }}$, $\mathrm{NF}^{\text {anti-miR-149 }}$ and NF ${ }^{\text {anti-NC }}$ were detected by qRT-PCR. (H) Concentration of IL-6 in the media of cultured CAF ${ }^{\mathrm{NC}}$, CAF ${ }^{\mathrm{miR}-149^{\prime}}$, $\mathrm{NF}^{\text {anti-miR-149 }}$ and NF ${ }^{\text {anti-NC }}$. (I, J) Relative FAP levels in CAF ${ }^{\text {miR-149 }}$ and NF ${ }^{\text {anti-miR-149 }}$ in the presence or absence of IL-6 or IL-6 Ab were analyzed by flow cytometry $(\mathrm{I})$ and $\mathrm{qRT}-\mathrm{PCR}(\mathrm{J})$. All data represent means $\pm \mathrm{SD}$ of three independent experiments. ${ }^{*} P<$ $0.05,{ }^{* *} P<0.01$, student's $t$-test. 
tissues and matched normal tissues, respectively. Quantitative analysis showed that miR-149 expression levels were markedly lower in GC CAFs than in NFs (Figure 1C).

The secretion of IL-6 was compared between CAFs and NFs, which showed higher levels of IL-6 secretion from CAFs than from NFs (Figure 1D). Moreover, IL-6 secretion levels were inversely correlated with miR-149 levels (Figure 1E). Taken together, these results suggest that miR-149 targets IL-6 mRNA and inhibits IL-6 production in CAFs.

miR-149 inhibits the activation of fibroblasts by reducing IL-6 expression

Fibroblast-derived IL-6 is critical for CAF function $[16,17]$, which prompted us to examine whether miR149 modulates CAFs through IL-6. We used the cell surface marker fibroblast activation protein (FAP) to evaluate the activation of fibroblasts. After confirming that FAP expression levels were higher in CAFs than in NFs by flow cytometry and mRNA quantification (Supplementary information, Figure S3A and S3B), the effect of miR-149 on FAP expression was examined by introducing miR-149 mimics and miR-149 inhibitors into CAFs and NFs, respectively. FAP expression was significantly downregulated by miR-149 mimics in CAFs and upregulated by miR-149 inhibitors in NFs (Figure $1 \mathrm{~F}$ and $1 \mathrm{G}$ ) similar to IL-6 secretion (Figure 1H). Addition of IL-6 or an IL-6 neutralizing antibody reversed the downregulation of FAP expression by miR-149 mimics and its upregulation by miR-149 inhibitors, respectively (Figure $1 \mathrm{I}$ and $1 \mathrm{~J}$ ). These results strongly support the notion that miR-149 plays a role in maintaining NFs and repressing the function of CAFs via the regulation of IL-6 expression.

miR-149 is critical for the tumor-promoting ability of fibroblasts

CAFs promote cancer cell proliferation, migration and invasion [1,31]. To determine whether miR-149 regulates the tumor-promoting ability of fibroblasts, we investigated the effects of conditioned media from CAFs or NFs with manipulated levels of miR-149 on GC cells (Figure 2A). As shown in Figure 2B-2E, miR-149 mimics significantly suppressed the stimulatory effect of CAFs on GC cell proliferation, colony forming ability, migration and invasion; conversely, miR-149 inhibitors conferred NFs the enhancing effects.

To further examine the role of miR-149 in vivo, nude mice were inoculated with SGC-7901 GC cells together with CAFs or NFs transfected with agomir-149 or antagomir-149 or their respective controls. As shown in Figure
2F-2H, miR-149 mimics significantly inhibited tumor growth and tumor burden in the group co-injected with GC cells and CAFs, whereas miR-149 inhibitors significantly increased tumor growth and tumor burden in the group co-injected with GC cells and NFs. The proliferation status of cells in tumors was verified by analyzing the proliferation marker Ki-67. The proliferation rate of cells positive for Ki-67 was dramatically reduced by miR-149, whereas it was substantially enhanced by the miR-149 inhibitor (Figure 2I and 2J). Taken together, our results indicate that miR-149 is an important negative regulator of the pro-tumorigenesis activity of CAFs both in vitro and in vivo.

CAFs enhance epithelial-to-mesenchymal transition and stem-like properties of GC cells in a miR-149-IL-6-dependent manner

Since CAFs stimulate epithelial-to-mesenchymal transition (EMT), which confers metastatic and self-renewal potential to cancer cells [36], and stem cell compartmentation during tumorigenesis [37], we postulated that miR-149 levels in CAFs might influence the EMT of GC cells. To prove this hypothesis, GC cells were treated with conditioned media from CAFs and NFs, and the expression of the epithelial cell marker E-cadherin and the mesenchymal cell markers N-cadherin, Vimentin and Snail was analyzed in GC cells. Conditioned medium from CAFs, but not that from NFs, induced the change of expression of EMT markers at the mRNA level (Figure 3A). An IL-6 neutralizing antibody blocked the CAF conditioned medium-induced the change of expression of EMT markers, whereas IL- 6 conferred the NF conditioned medium the ability to induce EMT, suggesting that IL-6 is crucial to this process (Figure $3 \mathrm{~A}$ ). Next, we grew SGC-7901 cells in different conditioned media from CAFs transfected with either miR-149 mimics or control and examined the expression of EMT markers at both mRNA and protein levels. miR-149 suppressed the CAF-induced EMT of GC cells, and the suppression was rescued by IL-6 (Figure 3B and 3C). The results of the culture of SGC-7901 cells using conditioned media from NFs transfected with either miR-149 inhibitors or control further supported the role of miR-149 and IL-6 in EMT induced by CAFs (Figure 3D and 3E). Furthermore, these results were confirmed by immunofluorescence detection of EMT markers in SGC-7901 cells cultured in different conditioned media (Figure $3 \mathrm{~F}$ and $3 \mathrm{G}$ ).

Cancer stem cells (CSCs) are considered as TICs that drive tumor formation, and the role of tumor stromal IL-6 in promoting the "stemness" of cancer cells has been established [38]. GC TICs are enriched in aldehyde dehydrogenase high [39] and side population (SP) cells 
A

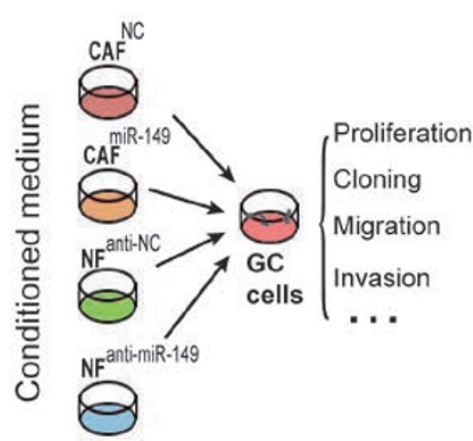

B

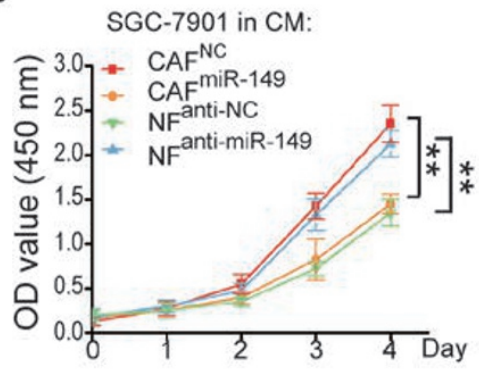

C

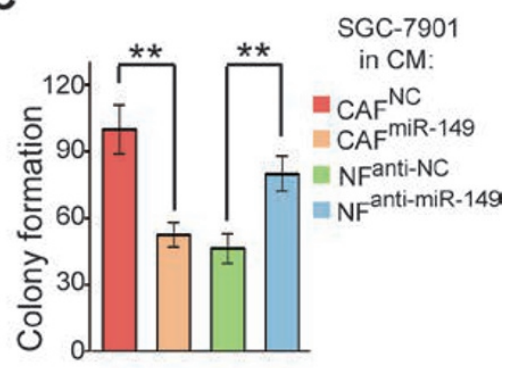

D
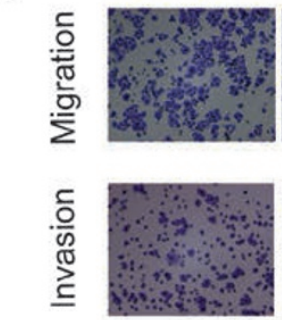

SGC-7901 CAF $^{\mathrm{NC}}$ in $\mathrm{CM}$ :
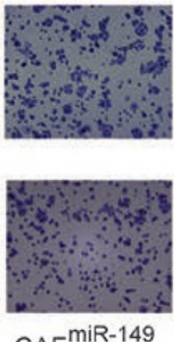

$\mathrm{CAF}^{\mathrm{miR}-149}$
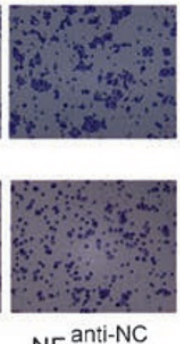

$\mathrm{NF}^{\text {anti-NC }}$
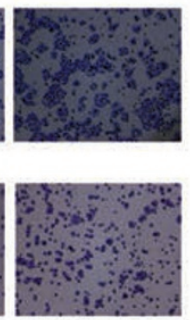

$\mathrm{NF}^{\text {anti-miR-149 }}$
E

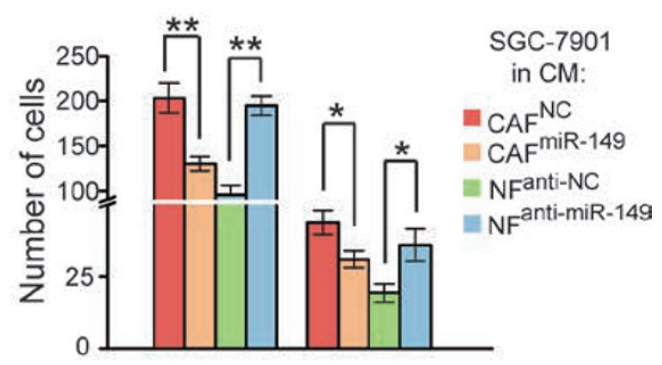

F

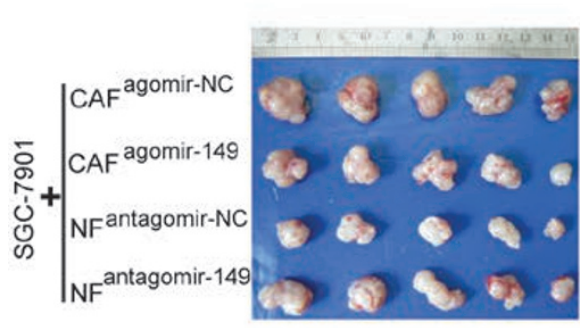

\section{G}

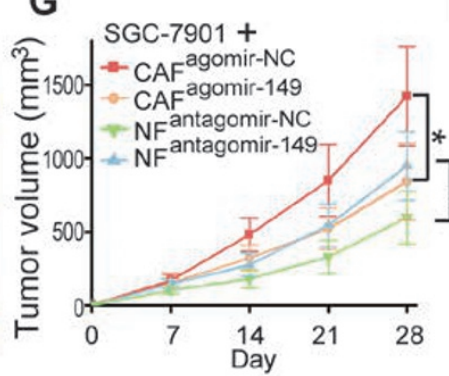

H

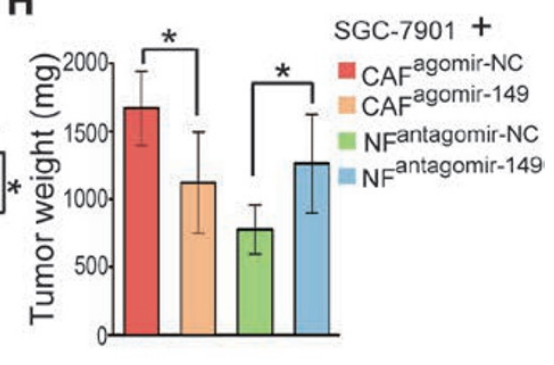

I

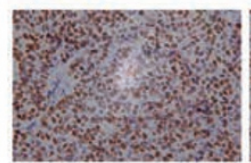

SCG7901

$+\mathrm{CAF}^{\text {agomir-NC }}$

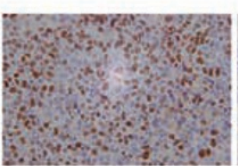

SCG7901

+ CAF $^{\text {agomir-149 }}$

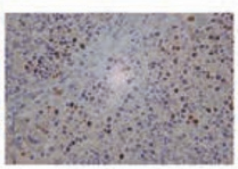

SCG7901

$+\mathrm{NF}^{\text {antagomir-NC }}$

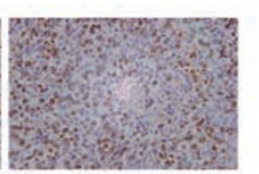

SCG7901

$+\mathrm{NF}^{\text {antagomir-149 }}$
J

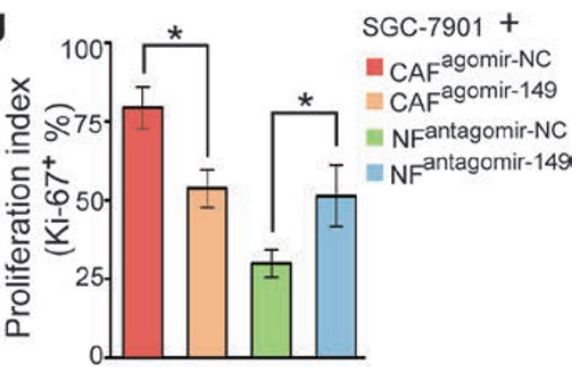

Figure 2 miR-149 influences the tumor-promoting ability of fibroblast. (A) Schematic chart of the evaluation of gastric cancer cell line SGC-7901 cultured in different conditioned medium (CM) as indicated. (B) Effect of miR-149 on GC cell proliferation measured by the WST assay. SGC-7901 cells were cultured in the conditioned mediums from CAF transfected with either miR-149 mimics or NC mimics, or NF transfected with either anti-miR-149 or anti-NC at a final concentration of $100 \mathrm{nM}$. (C) Effect of miR-149 on GC cell proliferation evaluated by colony formation assay. SGC-7901 cells were cultured in the indicated conditioned media and the number of colonies was counted 14 days after seeding. (D) Representative images of migratory or invasive cells on the membrane (magnification 100×). (E) Average number of migratory or invasive cells counted from $\mathbf{D}$. (F) Tumors derived from SGC-7901 cells mixed with different fibroblasts (CAF transfected with agomir-149 or agomir-NC, NF transfected with antagomir-149 or antagomir-NC) and injected subcutaneously into nude mice. Tumors were isolated 28 days after injection. (G) Growth kinetics of tumors in nude mice. Tumor diameters were measured and the volumes were calculated every 7 days. (H) Average weight of tumors from nude mice. (I) Representative images of tumor samples stained with Ki-67 antibody from nude mice (magnification 200x). (J) Relative proliferation index from I. All data are means \pm SD of three independent experiments. ${ }^{*} P<0.05,{ }^{* *} P<0.01$, student's $t$-test. 


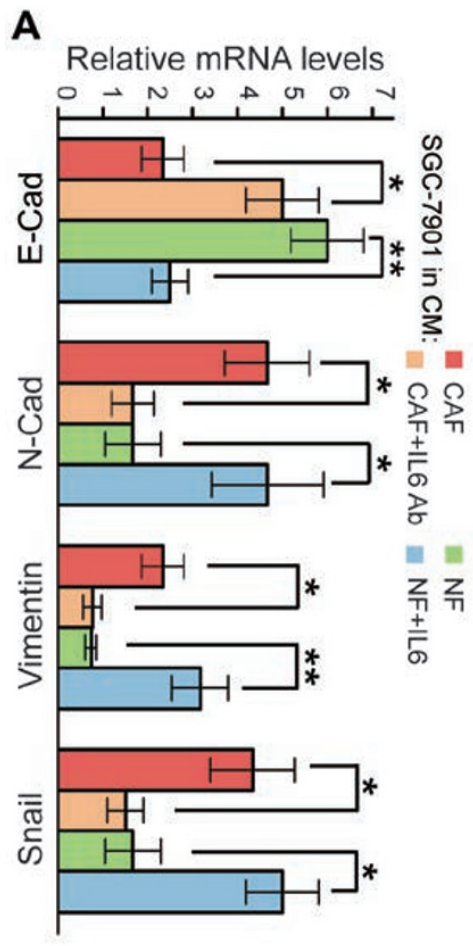

B

SGC-7901 in CM: $=\mathrm{CAF}^{\mathrm{NC}}$

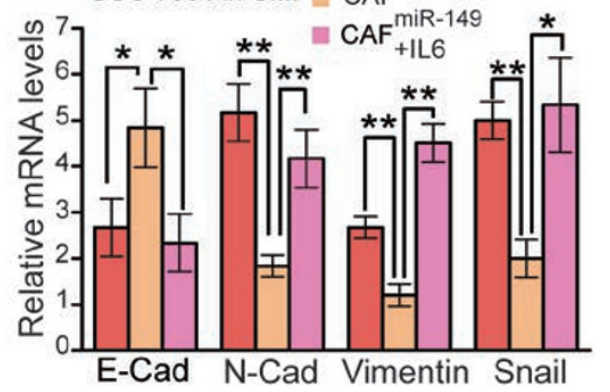

D<smiles>[N-]=[N-]</smiles>

SGC-7901 in CM: NF $^{\text {anti-miR-149 }}$

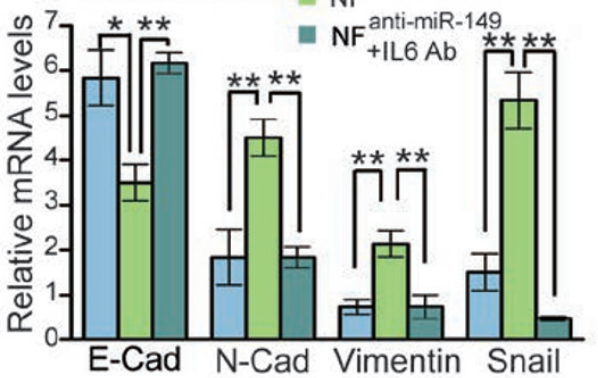

C

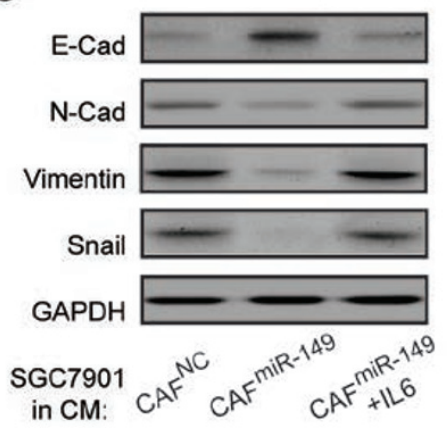

E

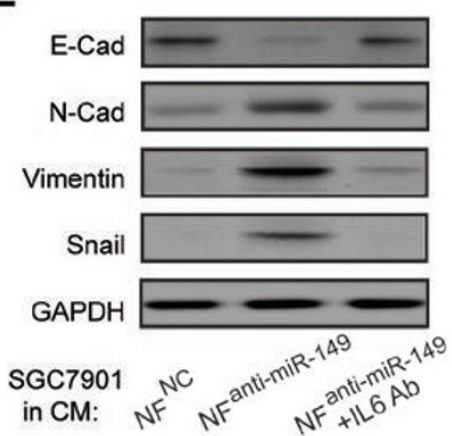

$\mathbf{F}$

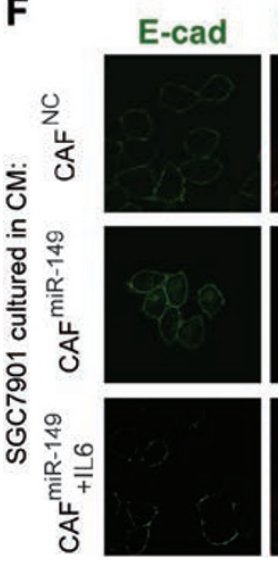

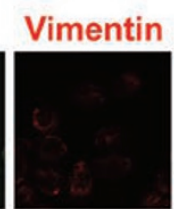
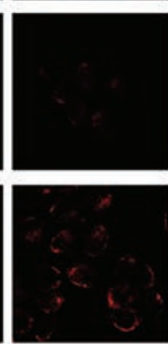
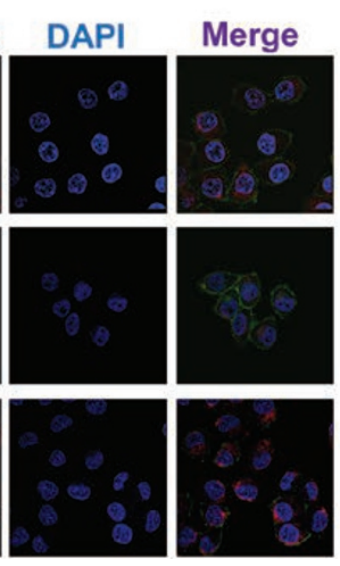

G

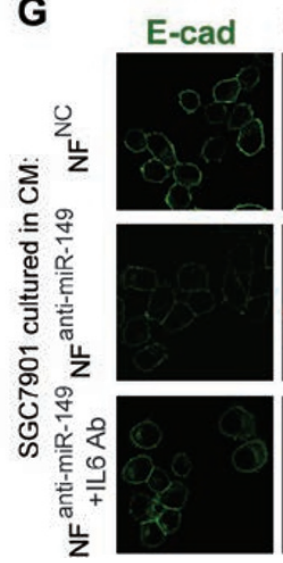

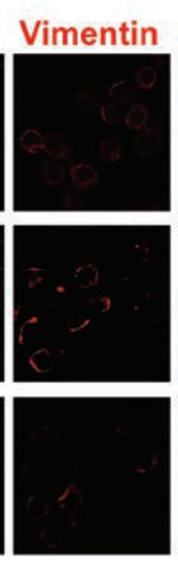

Merge
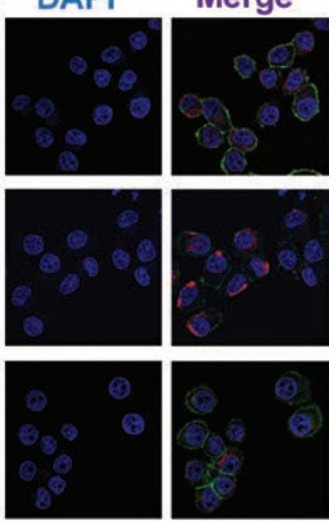

Figure 3 miR-149 inhibits the EMT of GC cells through IL-6. (A) Relative mRNA levels of EMT markers (E-cadherin, N-cadherin, Vimentin and Snail) in SGC-7901 cultured in the CM from CAF or NF in the presence or absence of IL-6 or IL-6 antibody as indicated were quantified by qRT-PCR. (B) Relative mRNA levels of EMT markers in SGC-7901 cultured in the CM from $\mathrm{CAF}^{\mathrm{NC}}$ or $\mathrm{CAF}^{\mathrm{miR}-149}$ in the presence or absence of IL-6 as indicated were quantified by qRT-PCR. (C) Protein levels of EMT markers in SGC-7901 cultured as in B were analyzed by western blotting. (D) Relative mRNA expression of EMT markers in SGC-7901 cultured in the CM from NF ${ }^{\text {anti-NC }}$ or NF ${ }^{\text {anti-miR-149 }}$ in the presence or absence of IL-6 antibody as indicated were quantified by qRT-PCR. (E) Protein levels of EMT markers in SGC-7901 cultured as in D were detected by western blotting. (F, G) Immunofluorescence analyses were carried out in SGC7901 cultured in the CM as indicated in B or D with antibodies for E-cadherin (green), Vimentin (red), and DAPI (blue), (magnification 400x). All data are means \pm SD of three independent experiments. ${ }^{*} P<0.05,{ }^{* *} P<0.01$, student's $t$-test.

[40]. We therefore investigated whether CAFs could increase the CSC-enriched population. We cultured GC cells with different conditioned media from CAFs and
NFs transfected with miR-149 mimics, miR-149 inhibitors or their respective controls, and then quantified the ALDH-positive or SP cells in GC cells by flow cytome- 
try. The proportions of cells with high levels of ALDH ${ }^{\text {high }}$ and SP cells were modulated by different conditioned media. As shown in Figure 4A, introduction of miR-149 into CAFs reduced the $\mathrm{ALDH}^{\text {high }}$ proportion of cancer cells cultured in conditioned medium from CAFs, and this was rescued by IL-6 supplementation. By contrast, anti-miR-149 boosted the ALDH ${ }^{\text {high }}$ proportion of cancer cells cultured in conditioned medium from NFs, and this was reversed by IL-6 neutralizing antibody (Figure 4C). A similar effect on the distribution of SP cancer cells was observed (Figure 4B and 4D). We also examined the effects of conditioned media from fibroblasts with manipulated levels of miR-149 on sphere formation of GC cells. As shown in Figure 4E and 4F, high miR-149 levels in CAFs had a negative effect on GC cell sphere formation and growth, and this was rescued by IL-6 supplementation, whereas the presence of anti-miR-149 in NFs had an enhancing effect that could be suppressed by an IL-6 neutralizing antibody. These results indicate that miR-149 in CAFs or NFs has an indirect effect on the proliferation of CSC or CSC-like cells.

PGE2 induces the epigenetic silencing of miR-149 in $\mathrm{fi}$ broblasts

DNA hypermethylation of many miRNAs is correlated with reduced gene expression in tumor tissues [41]. We identified an H3K27ac-enriched area [42] in a GC-rich region within -2500 to $1500 \mathrm{bp}$ upstream of the miR149 transcriptional start site using the MethPrimer-Tools and Databases (http://www.urogene.org/methprimer) and the ENCODE project data on the UCSC genome browser (http://goo.gl/6tDJ3v, Supplementary information, Figure S4A). We hypothesized that the downregulation of miR149 in CAFs could be associated with hypermethylation of this enhancer region. Methylation specific PCR (MSP) and bisulfite sequencing PCR (BSP) primers overriding this region were designed to detect the methylation status in CAFs and NFs. MSP analysis showed that this region was hypermethylated in CAFs compared to NFs (Supplementary information, Figure S4B). BSP analysis results showed that the average rate of $\mathrm{CpG}$ sites methylation was significantly higher in CAFs $(51.4 \% \pm 8.82 \%)$ than in NFs $(21.8 \% \pm 6.11 \%$; Figure 5A and Supplementary information, Figure S4C). These results suggest that hypermethylation of the miR-149 promoter is associated with the CAF phenotype and may contribute to the activation of fibroblasts.

PGE2 signaling is critical for IL-6 production in the tumor stroma and plays important roles in cancer and inflammation $[7,13]$. In addition, PGE2 promotes global methylation in tumor cells [43]. We postulated that PGE2 might be the signal triggering miR-149 methylation in fibroblasts. To test this possibility, we analyzed miR149 expression in NFs in response to PGE2 treatment and confirmed that PGE2 induced DNA methylation of miR-149 (Supplementary information, Figure S5) and downregulated miR-149 expression, while an inhibitor of DNA methylation, 5-Aza, abolished this effect (Figure $5 \mathrm{~B})$.

PGE2 receptor, PTGER2, is also a potential target of miR-149

We found that the PGE2 receptor, prostaglandin E receptor 2 (PTGER2, subtype EP2), also contains a seed match for miR-149 on its 3'-UTR (Figure 5C), and PGE2 can induce IL-6 expression in fibroblasts through EP2 [44]. We therefore, cloned the wild-type or mutant 3'UTR fragment of EP2 into the pMIR-REPORT luciferase vector (Supplementary information, Figure S6), and transfected the constructs together with miR-149 mimics or miR-149 inhibitor into fibroblasts, followed by luciferase reporter assay. The relative luciferase activity of pMIR/ $E P 2-3^{\prime}-\mathrm{UTR}^{\mathrm{wt}}$, but not that of $\mathrm{pMIR} / E P 2-3^{\prime}-\mathrm{UTR}^{\mathrm{mut}}$, was significantly suppressed by miR-149 mimics compared to NC mimics. By contrast, miR-149 inhibitors enhanced the activity of wild-type but not that of the mutant reporter (Figure 5D). This indicates that EP2 could be another potential target of miR-149. We therefore compared the EP2 expression levels in CAFs and NFs and found that it was higher in CAFs than in NFs (Supplementary information, Figure S7A) and the EP2 expression might be regulated by miR-149 (Supplementary information, Figure S7B). Furthermore, we also noticed higher EP2 expression level in tumor stroma compared to para-tumor normal stroma in the clinical specimens (Supplementary information, Figure S8).

Taken together, our data suggest a pro-tumor signaling mechanism by which stromal PGE2 induces IL-6 expression through EP2 by suppressing a shared inhibitory factor, miR-149 (Figure 5E). miR-149 targets IL-6 and EP2, downregulating their expression in fibroblasts. PGE2 binds to EP2 and induces the hypermethylation and suppression of miR-149, reversing the downregulation of IL-6 and EP2 expression.

\section{H. pylori infection activates $C O X-2 / P G E 2$ signaling in gastric epithelial cells \\ H. pylori infection may cause gastritis with a chron- ically inflamed stroma and the infection is recognized as the major cause of stomach cancer [45]. In addition, the COX-2/PGE2 pathway is activated upon $H$. pylori infection $[46,47]$. Thus, we hypothesized that $H$. pylori infection might elicit the malignant transformation of gastric epithelial cells, leading to a pro-inflammatory}


A

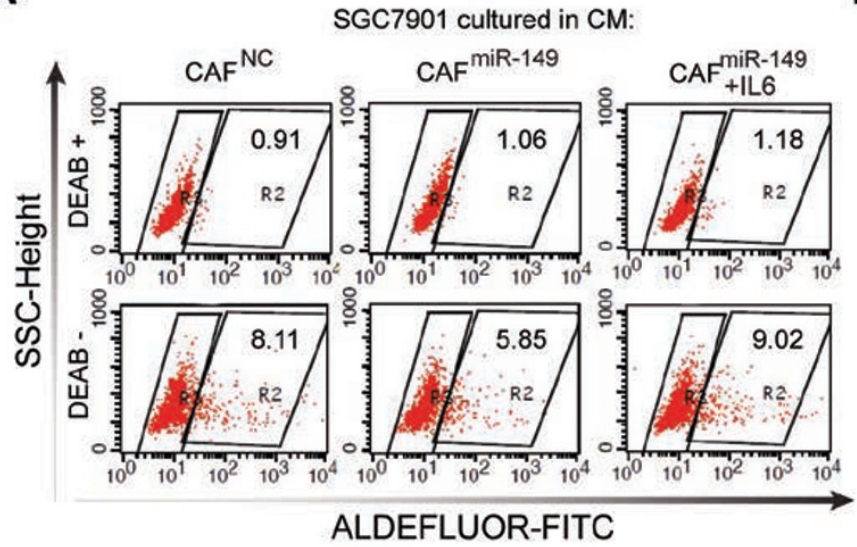

C

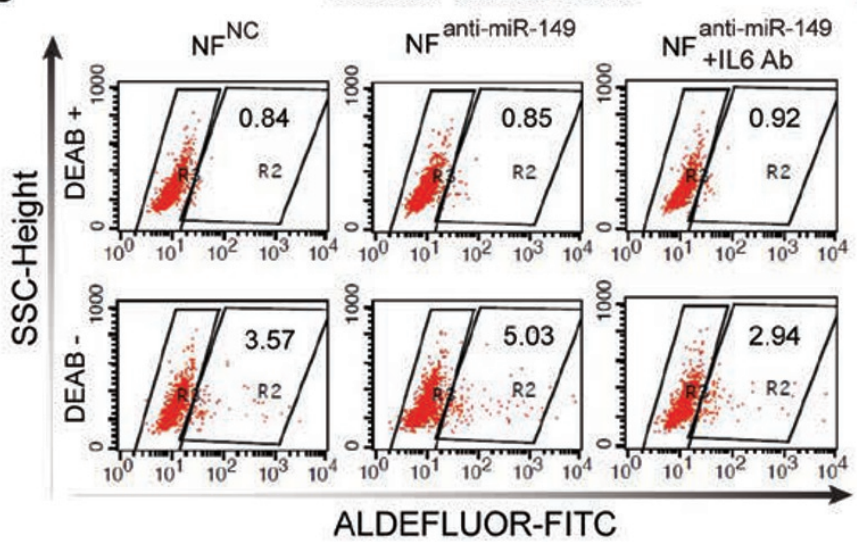

$\mathbf{E}$
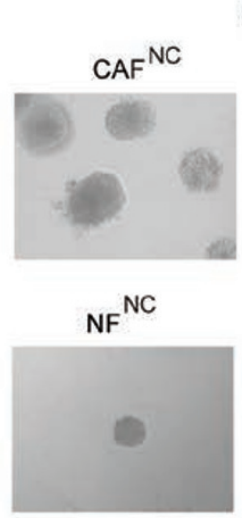

SGC7901 cultured in CM:
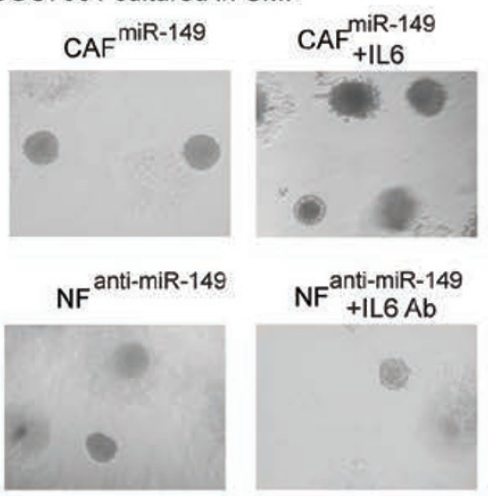

B

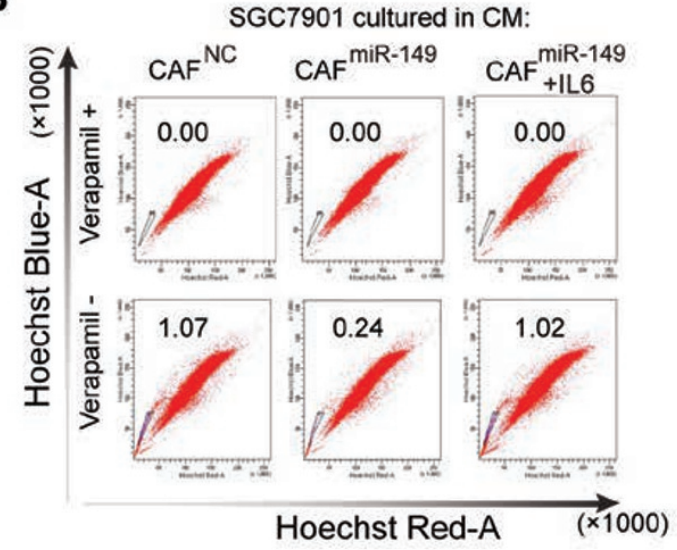

D

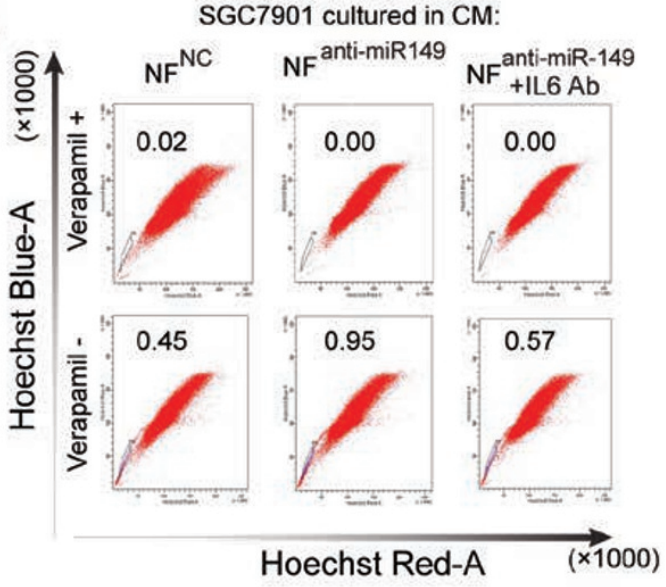

$\mathbf{F}$
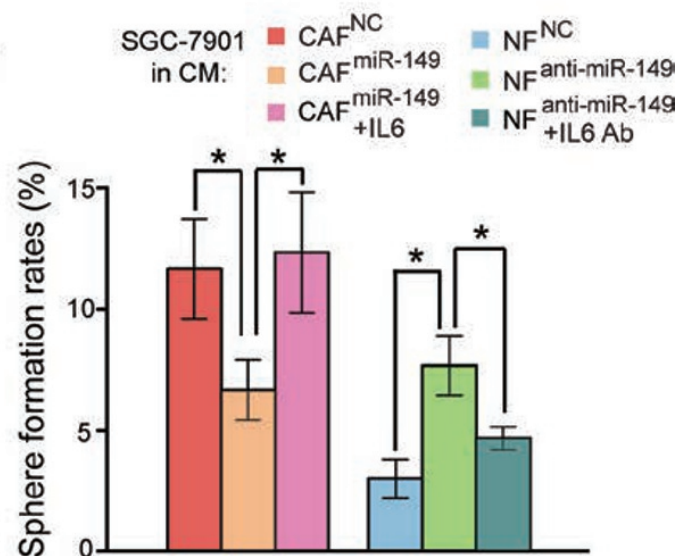

Figure 4 miR-149 inhibits the stem-like characteristics of GC cells through IL-6. (A) Aldeflour assay by flow cytometry of ALDH ${ }^{\text {high }}$ cells in SGC-7901 cells cultured in $\mathrm{CM}$ from CAF ${ }^{\mathrm{NC}}$ or $\mathrm{CAF}^{\text {miR-149 }}$ in the presence or absence of IL-6 as indicated. Representative flow cytometric analysis was shown. (B) Side population (SP) cells in SGC-7901 cells cultured as in A were measured by flow cytometry. SGC-7901 cells were stained with Hoechst 33342 dye in the presence (upper panel) or absence (bottom panel) of verapamil. Representative flow cytometric analysis was shown. (C) ALDH ${ }^{\text {high }}$ cells in SGC-7901 cells cultured in the $\mathrm{CM}_{\text {from }} \mathrm{NF}^{\text {anti-NC }}$ or $\mathrm{NF}^{\text {ant-miR-149 }}$ in the presence or absence of IL- 6 antibody as indicated by flow cytometric analysis. (D) SP cells in SGC-7901 cells cultured in the CM as in C and analyzed as in B. (E) Representative images of spheroid body from SGC-7901 cells cultured as in $\mathbf{A}$ or $\mathbf{C}$ (magnification 100x). (F) The rates of sphere formation were quantified. All data are means $\pm \mathrm{SD}$ of three independent experiments. ${ }^{*} P<0.05$, ${ }^{* \star} P<0.01$, student's $t$-test. 
A

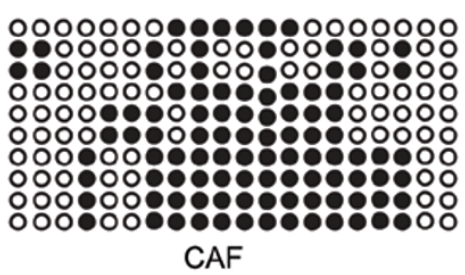

CAF

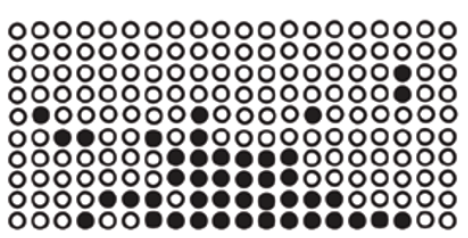

NF
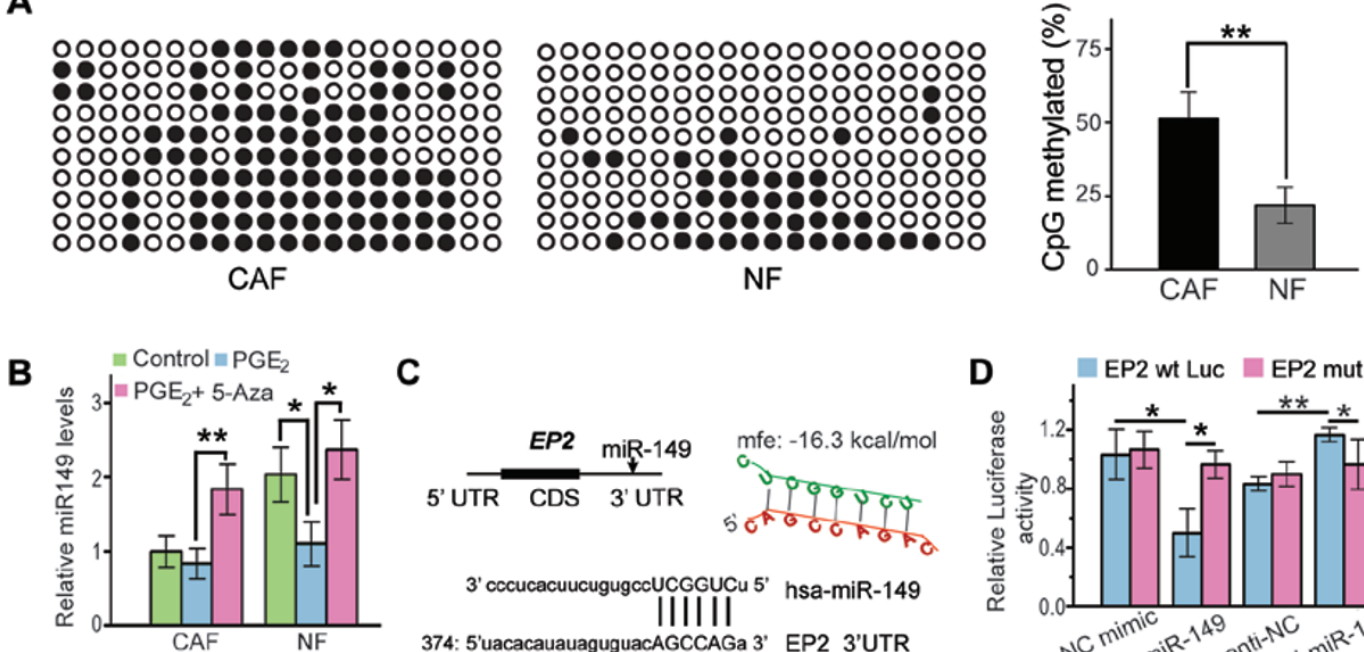

C

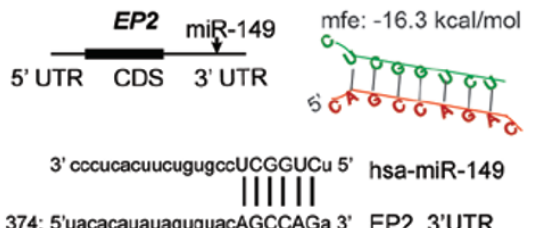

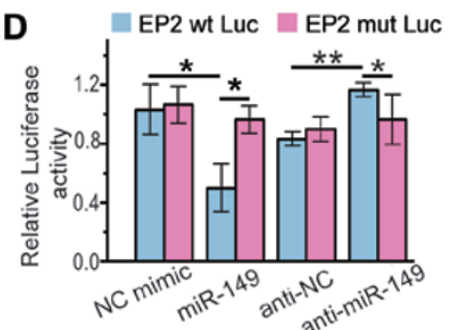

E
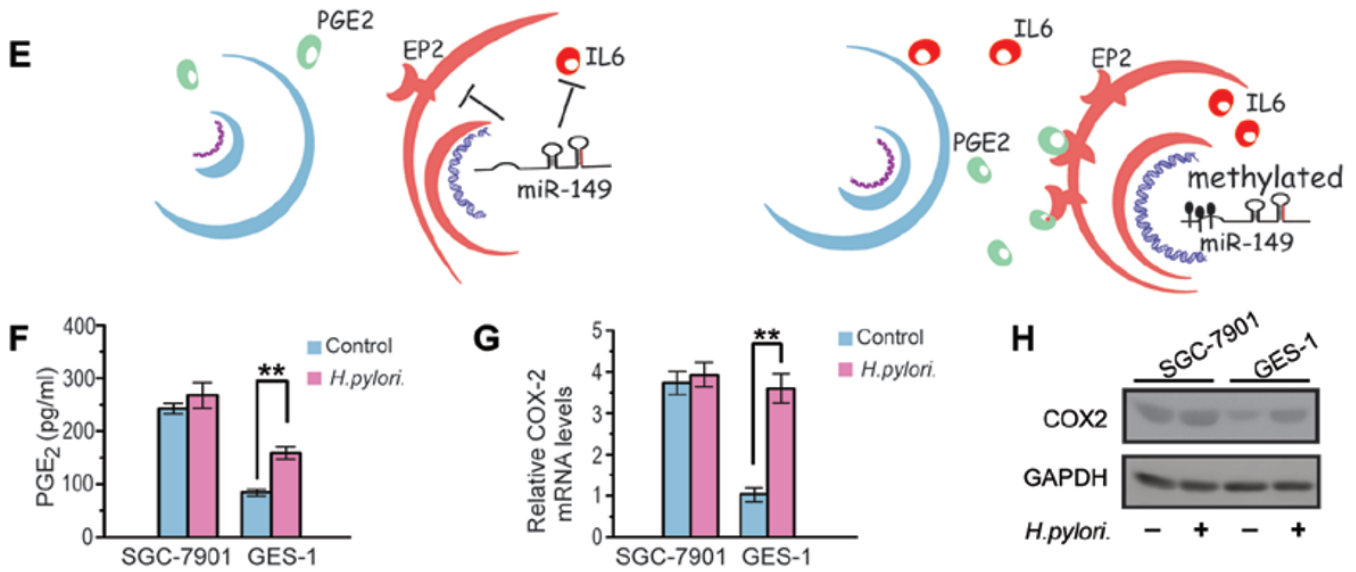

G
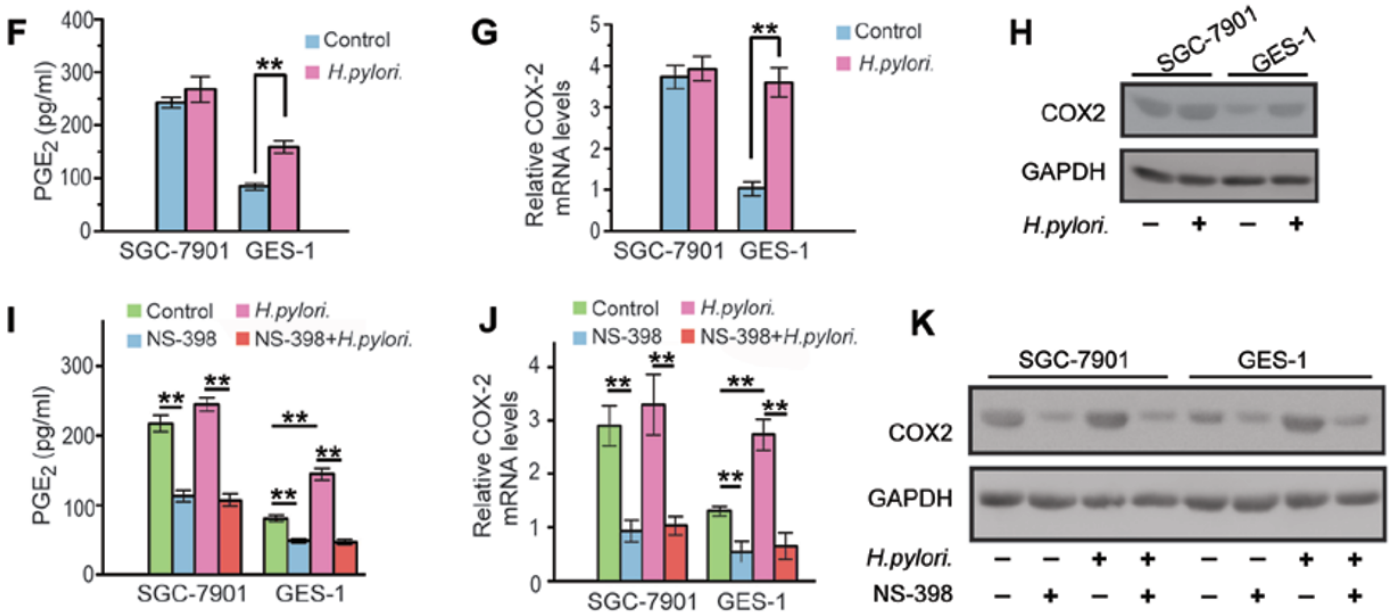

Figure $5 \mathrm{H}$. pylori-induced COX-2/PGE2 activation enhances epigenetic silencing of miR-149. (A) Methylation levels in the CpG sites rich region of miR-149 are higher in CAFs. Bisulfite sequencing was performed in 10 clones for each cell line. Open circle: unmethylated $\mathrm{CpG}$ site; filled circle: methylated $\mathrm{CpG}$ site. The ratios of methylation were summarized in the right panel. (B) miR-149 mRNA levels in CAF or NF treated or untreated with PGE2 or 5-Aza were analyzed by qRT-PCR. 5-Aza derepresses the PGE2-induced miR-149 repression. (C) Schematic graph of the 3'-UTR of EP2 with the putative binding site of $\mathrm{miR}-149$. The mfe predicted by RNAhybrid is $-16.3 \mathrm{kcal} / \mathrm{mol}$. (D) Relative luciferase activity in fibroblasts co-transfected with reporter vector with wild-type or mutant 3'-UTR of EP2 and miR-149 mimics, inhibitors or controls as indicated. (E) Sketch map of PGE2 activating IL-6 through EP2 by eliminating miR-149, a common inhibitory factor of IL-6 and EP2. miR149 targets IL-6 and EP2 for suppression in NFs (left panel). Through binding to EP2, PGE2 induces the hypermethylation and suppression of miR-149 that leads to the derepression of IL-6 and EP2 (right panel). (F) The PGE2 produced by SGC7901 and GES-1 cells with or without $H$. pylori infection was measured by ELISA. (G, H) COX-2 mRNA and protein levels in SGC-7901 and GES-1 cells as in F. mRNA and protein levels were analyzed by qRT-PCR and western blotting, respectively. (I) The PGE2 produced by SGC-7901 and GES-1 cells with or without $H$. pylori infection and in the presence or absence of NS-398 (50 $\mu \mathrm{mol} / \mathrm{L}$, an inhibitor of COX-2) was measured by ELISA. (J, K) COX-2 mRNA and protein levels in SGC-7901 and GES-1 cells as in I. mRNA and protein levels were analyzed by qRT-PCR and western blotting, respectively. All data are means \pm SD of three independent experiments. ${ }^{*} P<0.05$, ${ }^{* *} P<0.01$, student's $t$-test. 
response and consequently the dysfunction of miRNAs in fibroblasts, which in turn promotes tumor development. We infected cells with $H$. pylori and found that it induced PGE2 secretion more significantly in the gastric epithelial cell line GES-1 cells than in SGC-7901 cells (Figure 5F). Similarly, H. pylori infection induced COX2 expression at both protein and mRNA levels more significantly in GES-1 (Figure 5G and 5H). We used an inhibitor of COX-2, NS-398, and confirmed that it could block PGE2 secretion in both cell lines (Figure 5I). In addition, COX-2 expression at mRNA and protein levels was also inhibited by NS-398 in both GES-1 and SGC7901 cells with or without $H$. pylori infection (Figure 5J and $5 \mathrm{~K}$ ). These results indicate that $H$. pylori infection can activate the COX-2/PGE2 pathway to a greater extent in gastric epithelial cells than in GC cells.

H. pylori infection induces hypermethylation and downregulation of mmu-miR-149 in murine fibroblasts in vivo

$H$. pylori infection has been shown to be able to induce methylation-mediated silencing of miRNAs, which could be risk factors for $\mathrm{GC}[48,49]$. Our above results raised the possibility that $H$. pylori infection might activate COX-2/PGE2 signaling which in turn suppresses miR-149 expression through aberrant DNA methylation. To recapitulate human GC development in an animal model and to investigate whether $H$. pylori infection affects the hypermethylation and expression of the $m i R$ 149 gene in vivo, we performed intragastric administration of H. pylori in mice. The development of gastritis and $\mathrm{GC}$ in mice was verified by hematoxylin and eosin staining (Supplementary information, Figure S9B). Since the mouse $I L-6$ gene also contains a seed match for Mir149 (mmu-miR-149; Figure 6A), we performed a similar assay to that described in Figure 1 to confirm that miR149 also targets $I L-6$ in the mouse (Figure 6B and Supplementary information, Figure S9A). Fibroblasts from the stomach of control- and H. pylori-infected mice were isolated and cultured (Figure 6C). In those cells, Fap expression levels were higher in mouse CAFs (mCAFs) and mouse gastritis-associated fibroblasts (mGAFs) than in mouse NFs (mNFs; Supplementary information, Figure S9C). In addition, miR-149 levels were lower and IL-6 secretion levels were higher in both mCAFs and mGAFs than in mNFs (Figure 6D and 6E).

Subsequently, we analyzed the mouse ENCODE data and identified a $\mathrm{CpG}$ sites rich region between -2200 and $-1750 \mathrm{bp}$ upstream of the mmu-miR-149 that is conserved in humans (http://goo.gl/mqQu79, Supplementary information, Figure S9D). BSP analysis showed that the DNA methylation levels around that location were significantly higher in mCAFs $(45 \% \pm 3.7 \%)$ and
mGAFs $(47.3 \% 2.1 \%)$ than in mNFs $(8.3 \% \pm 2.9 \%$; Figure $6 \mathrm{~F}$ and $6 \mathrm{G}$, Supplementary information, Figure S9E). These results suggest that $H$. pylori infection is likely to promote the pro-tumor properties of stromal fibroblasts in vivo by silencing mmu-mir-149 and stimulating the production of IL-6.

\section{Discussion}

Although CAFs are not malignant themselves, they play a key role in the crosstalk between tumor cells and their microenvironment to support tumor growth $[1,37]$. In the present study, we identified miR-149 as a critical factor for the transformation of NFs into CAFs in GC. We showed that $H$. pylori infection activates the COX-2/ PGE2 pathway, leading to hypermethylation of the miR149 promoter and repression of miR-149 in human and mouse fibroblasts in vitro and in vivo. Through the regulation of IL-6 expression, suppression of miR-149 promotes the pro-tumor activity of CAFs by inducing EMT and stem-like traits in GC cells.

miRNAs have been implicated in the activation of fibroblasts, and they have been proposed as an emerging bridge linking $H$. pylori infection to $\mathrm{GC}[25,50]$. The up- and downregulation of miR-149 expression is associated with several types of cancer [51-55]. The existence of miRNA profiles specific to different cancers and even to certain subpopulations of cells in the same cancer is possible. We showed that miR-149 is significantly downregulated in CAFs from human GC tissues and H. pylo$r i$-infected mice (Figures 1 and 6), similar to the levels reported in breast CAFs [27]. This suggests the existence of a common mechanism underlying the regulation of CAFs in different cancers, which is further supported by the enhancement of IL-6 production by CAFs demonstrated in the present study (Figure 2) and in other reports [17-21].

The cytokine IL- 6 is required for the development of inflammation-associated cancers $[16,56]$. Increasing evidence strongly indicates that IL-6 is a common inflammatory factor secreted by CAFs from different sources $[3$, 15-18, 56-58]. A recent study showed that CAFs are activated in incipient neoplasia and contribute to tumor-promoting inflammation in an NF- $\mathrm{BB}-$ dependent manner [3]. In this process, $\mathrm{NF} \kappa \mathrm{B}$ acts as an essential positive regulator of IL-6. In the present study, we showed that miR-149 is downregulated in CAFs and acts as a negative regulator of IL-6 expression (Figure 1). Blocking the IL-6 signal using a neutralizing antibody has a similar negative effect to miR-149 upregulation on the activation of fibroblasts and the tumor-promoting ability of CAFs, whereas the inhibitory effect of miR-149 can be reversed 
A

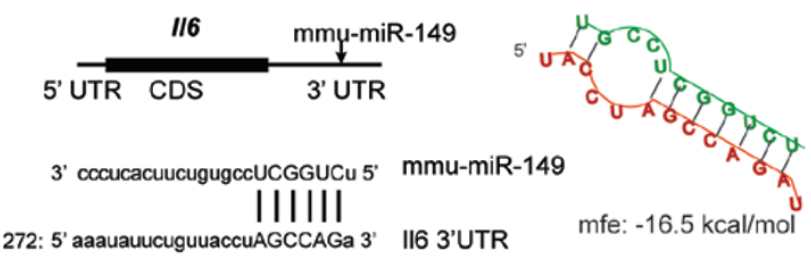

B

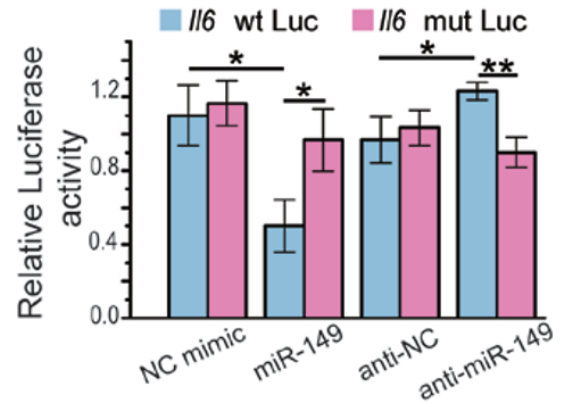

C

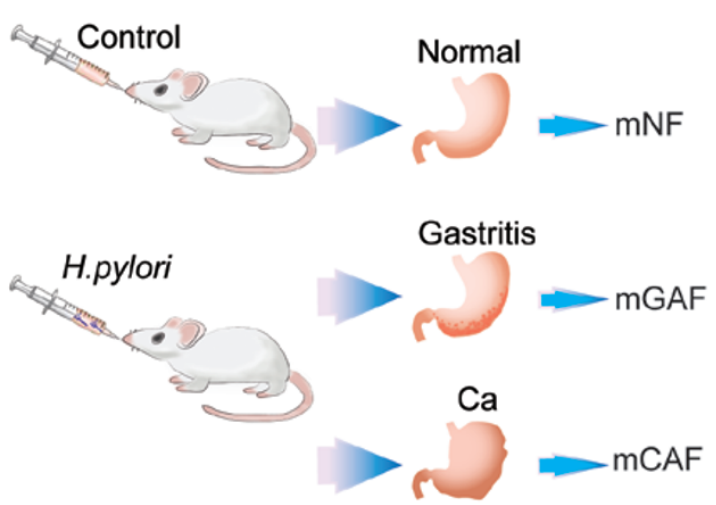

$\mathbf{F}$

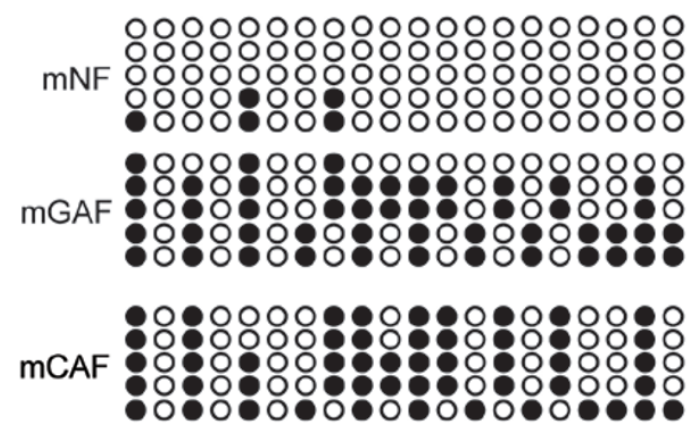

D

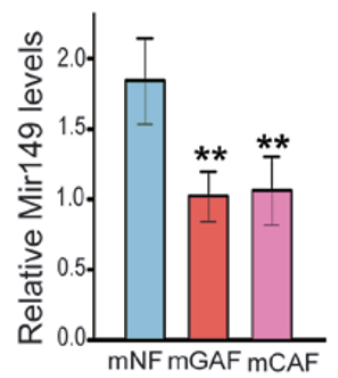

E

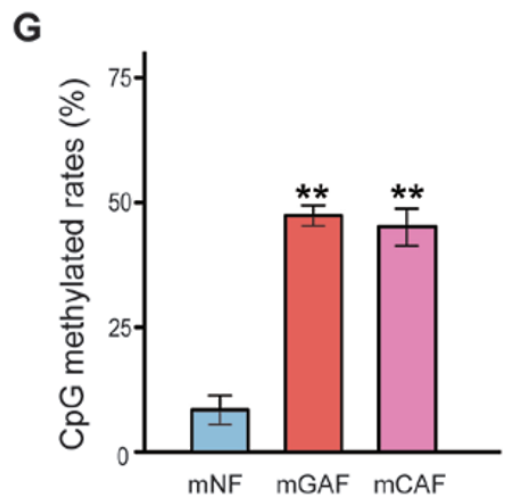

Figure 6 miR-149 is epigenetically silenced in fibroblasts from $H$. pylori-infected mouse stomach. (A) Schematic graph of 3'-UTR of IL-6 with the putative binding sites of mmu-miR-149. The mfe predicted by RNAhybrid is $-16.5 \mathrm{kcal} / \mathrm{mol}$. (B) Luciferase activity in cells co-transfected with reporter vector with wild-type or mutant IL-6 3'-UTR and mmu-miR-149 mimics or inhibitors as indicated. (C) Scheme for mice infected with $H$. pylori. Mouse normal fibroblasts (mNFs), mouse gastritis-associated fibroblasts (mGAFs) and mouse cancer-associated fibroblasts (mCAFs), were isolated from the control- and $H$. pylori-infected stomach of mice and cultured. (D) The mmu-miR-149 expression levels in mNFs, mGAFs and mCAFs were determined by qRT-PCR. (E) IL-6 secreted into media by mNFs, mGAFs and mCAFs was measured by ELISA. (F, G) Methylation levels in the $\mathrm{CpG}$ sites of miR-149 are higher in mCAF and mGAF than in mNF. Bisulfite sequencing was performed in 5 clones from each cell line. The rates of methylation were summarized in $\mathbf{G}$. All data are means \pm SD of three independent experiments. ${ }^{*} P<0.05,{ }^{* *} P<0.01$, student's $t$-test.

by supplementation of IL-6 (Figures 1 and 2). Our results indicate that miR-149 plays an important role in the activation of NFs and the maintenance of CAFs via the regulation of IL-6 expression, suggesting that epigenetic changes may guide the co-evolution of malignant cells and CAFs. During the preparation of this manuscript,
miR-149 was also found to be downregulated in osteoarthritis chondrocytes and it is correlated with increased expression of inflammatory cytokines including IL-6 [59]. Moreover, IL-6 is an important regulator of miRNA expression [60]. Therefore, IL-6 might be a pivot of miR-mediated inflammation in tumor microenvironment. 


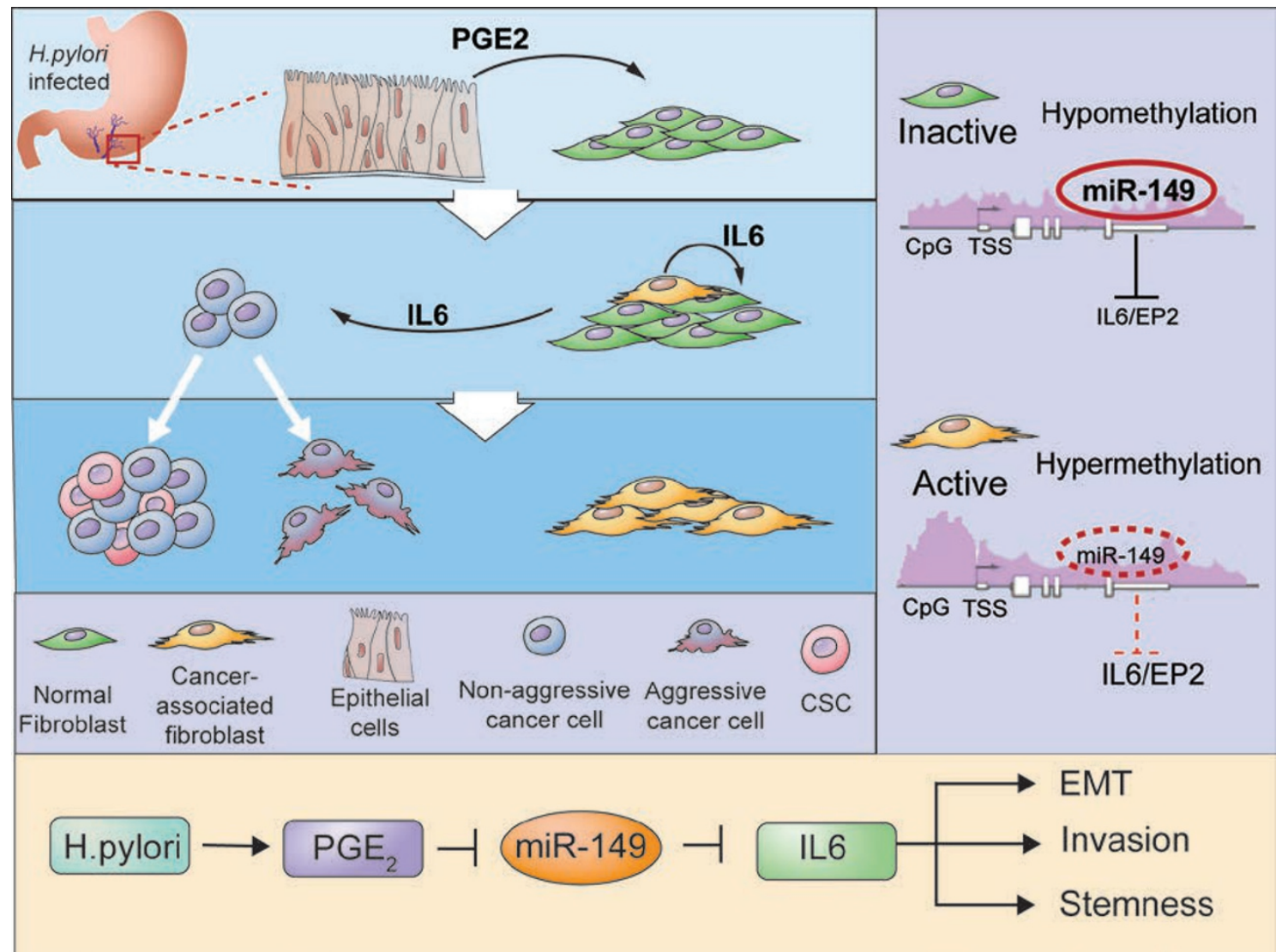

Figure 7 Schematic graph depicting the involvement of miR-149 in the interaction between $H$. pylori-infected gastric epithelial cells and gastric cancer-associated fibroblasts. $H$. pylori infection induces the secretion of PGE2 by gastric epithelial cells. Stromal PGE2 silences miR-149 probably through the hypermethylation of miR-149. The silence of miR-149 removes the suppression of its target genes, IL-6 and EP2, leading to elevated level of IL-6 that acts on fibroblasts to stimulate the transformation of NF into CAF. Meanwhile, IL-6 induces more aggressive behaviors of malignant gastric epithelial cells.

Tumor cells affect the secretion of IL-6 by stromal cells in the tumor microenvironment through mesenchymal stem cells (MSCs)-derived PGE2 [7]. The MSC-derived PGE2 operates in an autocrine manner to induce the production of IL-6, which together with PGE2 acts in a paracrine manner on tumor cells to induce EMT and increase the CSC population [7]. In the present study, PGE2 production was induced upon $H$. pylori infection in gastric epithelial cells (Figure 5F-5I). The nature of $H$. pylori-positive GC suggests that tumor cells originate from malignant transformed gastric epithelial cells, and $H$. pylori infection has been shown to induce the generation of CAFs in mice [15]. Therefore, our results support the notion that neoplastic PGE2 could be a trigger or early signal for the initiation of the transformation of NFs to CAFs [10].

DNA hypermethylation of many miRNAs is correlated with reduced gene expression in tumor tissues [61]. DNA hypermethylation of $m i R-149$ was detected in colorectal cancer [62]. In the present study, we showed the hypermethylation of a GC-rich region upstream of the miR149 transcriptional start site in CAFs from GC tissues, but not in NFs (Figure 5). Coincidently, PGE2 can induce the hypermethylation of miR-149 in fibroblasts (Figure S5) [43]. Surprisingly, we also found that the PGE2 receptor, EP2, is a target of miR-149. This indicates the existence of a positive feedback loop in CAFs in which PGE2 triggers the hypermethylation of $m i R-149$. The consequent silencing of miR-149 expression stimulates PGE2 signaling through the derepression of EP2. In the present study, the relationship between PGE2 and miR149 methylation (Figure 7) strengthens the argument that epigenetic modification is essential for the generation and maintenance of the CAF phenotype [30].

We investigated the role of $H$. pylori infection and miR-149 in a mouse model of gastritis and GC. Significantly lower miR-149 levels and correspondingly higher IL-6 secretion levels were detected in both mCAFs 
and mGAFs than in mNFs (Figure 6). Furthermore, we showed that $H$. pylori infection could activate the COX2/PGE2 pathway in gastric epithelial cells. PGE2 secretion and COX-2 expression are not apparently induced by $H$. pylori infection in GC SGC-7901 cells, and can be suppressed by the COX-2 inhibitor NS-398. This could be explained by the constitutive activation of the COX-2/ PGE2 signaling pathway in malignant epithelial cells.

Tumor development is dependent on the crosstalk between tumor cells and the tumor stroma. An understanding of how tumor cells communicate with the stroma will lead to the discovery of innovative avenues of therapeutic intervention. In the present study, we showed that miR-149 modulates PGE2 and IL-6 signaling in the crosstalk between tumor cells and the tumor stroma. These findings shed light on the mechanism of action of the PGE2-IL-6 activation loop and further highlight the significance of this loop in tumorigenesis. We propose that $H$. pylori infection-induced PGE2 secretion from epithelial cells or from tumor cells promotes the methylation of $m i R-149$ and the subsequent induction of the expression of its target gene (IL-6) in stromal fibroblasts. The role of miRNAs as a sensor signal triggered upon $H$. pylori infection in the mucosa and epithelium has been proposed [49]. However, our results support a role for miR-149 in stromal cells as a potential amplifier signal triggered by $H$. pylori infection. Therefore, detection or intervention of miR-149 expression in the tumor stroma could be of prognostic or therapeutic value in GC.

\section{Materials and Methods}

\section{Cell lines and primary cell isolation}

GC cell line SGC-7901 and the immortalized gastric epithelial cell line GES-1 were purchased from Shanghai Institutes for Biological Sciences, Chinese Academy of Sciences, and were cultured at $37{ }^{\circ} \mathrm{C}$ with RPMI- 1640 medium containing $10 \%$ fetal calf serum. Fibroblasts were isolated from five independent GC patients undergoing radical gastrostomy at the Department of Surgery, Ruijin Hospital, Shanghai Jiao Tong University. None of the patients received preoperative treatment. The primary fibroblast isolation and culture were described in our previous study [63]. The tumor tissues and non-tumor tissues were collected, and after cleaning and disinfection, the tissues were minced into organoids of $\sim 1$ $\mathrm{mm}^{3}$ and seeded into petri dishes. These conditions produced a homogenous fibroblastic cell population after about 7 days of culture. The doubling time of fibroblasts is about 2-3 days. We harvested cells passaged for up to 10 population doublings for subsequent experiments in order to maintain the characters and the phenotype of primary cells.

\section{Reagents}

Recombinant IL-6 was purchased from PeproTech. FAP antibody was purchased from Santa Cruz Biotech; E-cadherin, N-cadherin, Vimentin, Snail and COX-2 antibodies were purchased from
Abcam; EP2 antibody was purchased from BBI; Human IL-6 mAb and mouse IL- 6 mAb were purchased from R\&D Systems (Minneapolis, MN, USA).

\section{$R N A$ isolation and $q P C R$}

Total RNA was isolated using Trizol regent (Invitrogen, Carlsbab, CA, USA) or mirVanaTM miR Isolation Kit (Applied Biosystems, Foster City, CA, USA) according to the manufacturer's instructions. The mRNA expression level was measured by qPCR according to TaqMan Gene Expression Assays protocol (Applied Biosystems). The primers used were: FAP: 5'-AATGAGAGCACTCACACTGAAG-3' and 5'-CCGATCAGGTGATAAGCCGTAAT-3'; E-cadherin: 5'-CCCACCACGTACAAGGGTC-3' and 5'-CTGGGGTATTGGGGGCATC-3'; N-cadherin: 5'-CAACTTGCCAGAAAACTCCAGG-3' and 5'-ATGAAACCGGGCTATCTGCTC-3'; Vimentin: 5'-AAAACACCCTGCAATCTTTCAGA-3' and 5'-CACTTTGCGTTCAAGGTCAAGAC-3; Snail: 5'-CCCCAATCGGAAGCCTAACT-3' and 5'-GGTCGTAGGGCTGCTGGAA-3'; COX-2: 5'-TAAGTGCGATTGTACCCGGAC-3' and 5'-TTTGTAGCCATAGTCAGCATTGT-3'; Fap (Mus musculus): 5'-GGCTGGGGCTAAGAATCCG-3' and 5'-GCATACTCGTTCACTGGACAC-3. The expression levels of miRNAs were measured by miRNAs qPCR Kit (GenePharma, Shanghai, China) according to the manufacturer's instructions.

\section{Transfection of miR mimics}

Oligonucleotides for hsa-miR-149 mimics (miR-149), negative control (miR-NC), hsa-miR-149 inhibitors (anti-miR-149), inhibitor negative control (anti-miR-NC) and mmu-miR-149 mimics, inhibitors or controls were purchased from GenePharma (Shanghai, China). Transfection of cells with oligonucleotides was performed with Lipofectamine 2000 (Invitrogen) at a final concentration of $100 \mathrm{nM}$. For the cells used in tumor xenograft mouse model, chemically modified mimic (agomir) or antisense (antagomir) oligonucleotides, which were also from GenePharma, for hasmiR-149 was applied.

\section{Luciferase activity assay}

The fragment of wild-type (wt) 3'-UTR of IL-6 containing the putative miR-149-binding sites or mutant (mut) 3'-UTR of IL-6 were synthesized and cloned into the pMIR-Report luciferase vector (Ambion) containing Firefly, and they were named $I L-6-3^{\prime}-$ $\mathrm{UTR}^{\mathrm{wt}}$ Luc and $I L-6-3^{\prime}-\mathrm{UTR}^{\mathrm{mut}}$ Luc. Cells were seeded into the 24well plates $24 \mathrm{~h}$ before transfection. A total of $500 \mathrm{ng}$ of $I L-6-3^{\prime}-$ $\mathrm{UTR}^{\mathrm{wt}}$ Luc or $I L-6-3^{\prime}-\mathrm{UTR}^{\mathrm{mut}}$ Luc were transfected into each well together with $20 \mathrm{ng}$ of pRL-TK vector (Promega) and $60 \mathrm{pmol}$ of oligonucleotides. Cells were harvested $48 \mathrm{~h}$ later and Firefly and Renilla luciferase activities were measured by Dual-luciferase reporter assay (Promega). This method was also performed to verify that miR-149 targets the 3'-UTR of EP2 and mmu-mir-149 targets the 3'-UTR of IL-6 (Mus musculus).

\section{ELISA}

The levels of cytokines IL-6 and PGE2 in supernatants were detected by ELISA kit (R\&D Systems) according to the instructions of manufacturer.

\section{Cell proliferation assay}

Cell proliferation was assessed by WST (water-soluble tetra- 
zolium salt) assay using a Cell Counting Kit-8 (Dojindo, Japan) according to the manufacturer's instructions. Cells $\left(2 \times 10^{3}\right.$ cells/ well) were seeded into 96 -well plates. The cell proliferation was examined every $24 \mathrm{~h}$ for 5 days. The number of viable cells was assessed by measurement of the absorbance at $450 \mathrm{~nm}$.

\section{Soft agar colony formation assay}

Cells were resuspended with $0.3 \%$ soft agar in RPMI 1640 containing $10 \%$ FBS or conditioned medium and layered onto $0.6 \%$ solidified agar in RPMI1640 containing 10\% FBS in 6-well plates $\left(1 \times 10^{3}\right.$ cells/well). The plates were incubated at $37{ }^{\circ} \mathrm{C}$ in humidified atmosphere of $5 \% \mathrm{CO}_{2}$ for 2 weeks, the stained with $0.02 \%$ crystal violet for $1 \mathrm{~h}$ (Sigma, USA). The colonies were counted in 5 random fields under microscope. Colonies containing at least 50 cells were counted.

\section{Cell migration and invasion assay}

Migration of cells was performed using QCM 24-Well Colorimetric Migration Assay Kit (Millipore) according to the manufacturer's instructions. For the invasion assay, Cell Invasion Assay Kit (Millipore) was used. Cells $\left(2 \times 10^{4}\right)$ in $200 \mu 1$ serum-free medium were added to the upper chambers and cultured for $48 \mathrm{~h}$. Non-migrating or non-invading cells were removed with cotton swabs, cells that migrated or invaded to the bottom of the membrane were stained with the cell stain buffer and counted under microscope and photographed. Three independent experiments were performed for the same conditions.

\section{Tumor xenograft model and tumorigenicity assay}

SGC-7901 cells co-cultured with fibroblasts were subcutaneously injected into 4-week-old male nude mice (Institute of Zoology, Chinese Academy of Sciences). The mice were checked every 7 days, and the tumor nodules were measured with a caliper. Mice were euthanized 28 days after injection, the tumors were removed and weighed and tumor volume was evaluated. Tumor growth curves were calculated. All tumor grafts were excised, weighed, harvested, fixed and embedded. The Ki-67 antibody (Dako, dilution 1:100) was used to determine the expression of Ki-67 by immunohistochemistry. The proliferative index score was measured by the rate of Ki-67-positive cells.

\section{SP analysis}

The cells were removed, pelleted by centrifugation, washed with PBS (phosphate-buffered saline), and suspended at $1 \times 10^{6}$ cells $/ \mathrm{ml}$ in PBS supplemented with $4 \%$ FBS and incubated at $37^{\circ} \mathrm{C}$ for $60 \mathrm{~min}$ in the dark with the DNA binding dye, Hoechest $33342(5 \mu \mathrm{g} / \mathrm{ml}$, Sigma, USA), either alone or in the presence of verapamil (500 $\mu \mathrm{M}$, Sigma, USA). After incubation, $1 \mu \mathrm{g} / \mathrm{ml}$ propidium iodide was added and then the cell suspension was filtered through a $40 \mu \mathrm{m}$ cell strainer to obtain single suspension cells, and the cells were kept at $4{ }^{\circ} \mathrm{C}$ in the dark before FACS. Analyses and sorting were performed using BD FACS Aria II (BD, New Jersey, USA).

\section{Examination of ALDH activity by Aldeflour reagent}

Aldeflour assay kit (Stemcell Technologies, Vancouver, Canada) was used to detect the ALDH activity of cells. Cancer cells $\left(1 \times 10^{6}\right.$ cells $\left./ \mathrm{ml}\right)$ were suspended in Aldeflour assay buffer and incubated with the ALDH substrate (BODIPY-aminoacetaldehyde,
BAAA) for $45 \mathrm{~min}$ at $37{ }^{\circ} \mathrm{C}$. As a negative control, $1.5 \mathrm{mM}$ diethylaminobenzaldehyde (DEAB), an ALDH specific inhibitor was added. FACS Calibur flow cytometer was used for cell analysis.

\section{Confocal immunofluorescence}

The PathScan EMT Duplex IF Kit (Cell Signaling Technology) was adopted to detect the expression of the EMT markers: E-cadherin (Alexa Flour488) and Vimentin (Alexa Fluor555). Cells were fixed in $4 \%$ formaldehyde buffer for $15 \mathrm{~min}$ at room temperature. Cells were then blocked in blocking buffer for 60 min and incubated with the primary antibodies overnight at $4{ }^{\circ} \mathrm{C}$. After being rinsed three times with PBS, cells were incubated with detection cocktail buffer at room temperature in the dark, covered with slides with Antifade Reagent (with DAPI) and subjected to confocal microscopy analyses (LSM510, Carl Zeiss, Jena, Germany).

\section{DNA isolation and methylation analysis}

Genomic DNA from tissue samples was purified using DNAzol (Invitrogen). Sodium bisulfite conversion was conducted using the Qiagen Epitect Bisulfite Kit. The methylation status was examined by MSP or BSP. The MSP products were confirmed by agarose gel electrophoresis. The BSP products were subcloned into pMD19-T Vector (Takara) according to the manufacturer's instructions.

\section{Mice and sample preparation}

Following previously reported procedures [64-66], BALB/c mice (Institute of Zoology, Chinese Academy of Sciences) were divided into three groups: Group 1 and Group 2 of 20 mice each as the H. pylori-infected groups, Group 3 as the normal control group. Mice in Group 1 were inoculated with $0.1 \mathrm{ml}$ suspension of $H$. pylori SS1 strain $\left(1.0 \times 10^{9} \mathrm{CFU} / \mathrm{ml}\right)$ intragastrically every other day from age of 5 weeks, and were given distilled drinking water. Group 2 were inoculated with $H$. pylori as Group 1, and were treated with N-methyl-N-nitrosourea (MNU, Sigma-Aldrich) mixed in drinking water $(60 \mathrm{ppm}$, freshly prepared 3 times per week) for 10 weeks; Group 3 were given distilled water, but not treated with MNU and H. pylori. All mice were sacrificed 40 weeks after treatment.

The mouse stomach was resected and cut open along the greater curvature. Half of them were harvested, fixed and embedded. H\&E staining was performed to detect the H. pylori colonization and tumor occurrence. The other half was used for primary fibroblast culture.

\section{Statistical analysis}

Results were summarized as means $\pm \mathrm{SD}$. Student's $t$-test and one-way analysis of variance were used to analyze the data and the significance level was set at $P<0.05$.

\section{Acknowledgments}

We thank Dr Andrew J Dannenberg (Weill Cornell Medical College, USA) for suggestion and critical comments on this manuscript. This work was supported by grants from the National Natural Science Foundation of China (81172324, 81300290, 81372187, 91229106, 81272749), Science and Technology Commission of Shanghai Municipality (12XD1403700), Key Projects in the National Science \& Technology Pillar Program of China 
(2014BAI09B03), Key Project of Shanghai Education Committee (12ZZ105 and 12ZZ102) and China Postdoctoral Science Foundation (2011M500796).

\section{References}

1 Franco OE, Shaw AK, Strand DW, Hayward SW. Cancer associated fibroblasts in cancer pathogenesis. Semin Cell Dev Biol 2010; 21:33-39.

2 Orimo A, Gupta PB, Sgroi DC, et al. Stromal fibroblasts present in invasive human breast carcinomas promote tumor growth and angiogenesis through elevated SDF-1/CXCL12 secretion. Cell 2005; 121:335-348.

3 Erez N, Truitt M, Olson P, Arron ST, Hanahan D. Cancer-associated fibroblasts are activated in incipient neoplasia to orchestrate tumor-promoting inflammation in an NF- $\mathrm{BB}-\mathrm{de}-$ pendent manner. Cancer Cell 2010; 17:135-147.

4 Swartz MA, Iida N, Roberts EW, et al. Tumor microenvironment complexity: emerging roles in cancer therapy. Cancer Res 2012; 72:2473-2480.

5 Albini A, Sporn MB. The tumour microenvironment as a target for chemoprevention. Nat Rev Cancer 2007; 7:139-147.

6 Subbaramaiah K, Telang N, Ramonetti JT, et al. Transcription of cyclooxygenase-2 is enhanced in transformed mammary epithelial cells. Cancer Res 1996; 56:4424-4429.

7 Li HJ, Reinhardt F, Herschman HR, Weinberg RA. Cancer-stimulated mesenchymal stem cells create a carcinoma stem cell niche via prostaglandin E2 signaling. Cancer Discov 2012; 2:840-855.

8 Liu $\mathrm{CH}$, Chang SH, Narko K, et al. Overexpression of cyclooxygenase-2 is sufficient to induce tumorigenesis in transgenic mice. J Biol Chem 2001; 276:18563-18569.

9 Subbaramaiah K, Howe LR, Port ER, et al. HER-2/neu status is a determinant of mammary aromatase activity in vivo: evidence for a cyclooxygenase-2-dependent mechanism. Cancer Res 2006; 66:5504-5511.

10 Wang D, Dubois RN. Eicosanoids and cancer. Nat Rev Cancer 2010; 10:181-193.

11 Williams CS, Tsujii M, Reese J, Dey SK, DuBois RN. Host cyclooxygenase-2 modulates carcinoma growth. $J$ Clin Invest 2000; 105:1589-1594.

12 Ishikawa TO, Herschman HR. Tumor formation in a mouse model of colitis-associated colon cancer does not require COX-1 or COX-2 expression. Carcinogenesis 2010; 31:729736.

13 Hinson RM, Williams JA, Shacter E. Elevated interleukin 6 is induced by prostaglandin E2 in a murine model of inflammation: possible role of cyclooxygenase-2. Proc Natl Acad Sci USA 1996; 93:4885-4890.

14 Zhang Y, Lin JX, Vilcek J. Synthesis of interleukin 6 (interferon-beta $2 / B$ cell stimulatory factor 2 ) in human fibroblasts is triggered by an increase in intracellular cyclic AMP. J Biol Chem 1988; 263:6177-6182.

15 Quante M, Tu SP, Tomita H, et al. Bone marrow-derived myofibroblasts contribute to the mesenchymal stem cell niche and promote tumor growth. Cancer Cell 2011; 19:257-272.

16 Kinoshita H, Hirata Y, Nakagawa H, et al. Interleukin-6 mediates epithelial-stromal interactions and promotes gastric tumorigenesis. PLoS One 2013; 8:e60914.
17 Nagasaki T, Hara M, Nakanishi H, Takahashi H, Sato M, Takeyama H. Interleukin-6 released by colon cancer-associated fibroblasts is critical for tumour angiogenesis: anti-interleukin-6 receptor antibody suppressed angiogenesis and inhibited tumour-stroma interaction. Br J Cancer 2014; 110:469-478.

18 Paland N, Kamer I, Kogan-Sakin I, Madar S, Goldfinger N, Rotter V. Differential influence of normal and cancer-associated fibroblasts on the growth of human epithelial cells in and in vitro cocultivation model of prostate cancer. Mol Cancer Res 2009; 7:1212-1223.

19 Liu XH, Kirschenbaum A, Yao S, Levine AC. Interactive effect of interleukin- 6 and prostaglandin E2 on Osteoclastogenesis via the OPG/RANKL/RANK system. Ann N Y Acad Sci 2006; 1068:225-233.

20 Bouffi C, Bony C, Courties G, Jorgensen C, Noël D. IL-6-dependent PGE2 secretion by mesenchymal stem cells inhibits local inflammation in experimental arthritis. PLoS One 2010; 5:e14247.

21 Calin, GA, Croce CM. MicroRNA signatures in human cancers. Nat Rev Cancer 2006; 6:857-866.

22 Mitra AK, Zillhardt M, Hua Y, et al. MicroRNAs reprogram normal fibroblasts into cancer-associated fibroblasts in ovarian cancer. Cancer Discov 2012; 2:1100-1108.

23 Wang M, Li C, Nie H, et al. Downregulated miR-625 suppresses invasion and metastasis of gastric cancer by targeting ILK. FEBS Lett 2012; 586:2382-2388.

24 Li P, Chen X, Su L, et al. Epigenetic silencing of miR-338$3 \mathrm{p}$ contributes to tumorigenicity in gastric cancer by targeting SSX2IP. PLoS One 2013; 8:e66782.

25 Aprelikova O, Yu X, Palla J, et al. The role of miR-31 and its target gene SATB2 in cancer-associated fibroblasts. Cell $\mathrm{Cy}$ cle 2010; 9:4387-4398.

26 Enkelmann A, Heinzelmann J, von Eggeling F, et al. Specific protein and miR patterns characterise tumour-associated fibroblasts in bladder cancer. J Cancer Res Clin Oncol 2011; 137:751-759.

27 Zhao L, Sun Y, Hou Y, et al. MiRNA expression analysis of cancer-associated fibroblasts and normal fibroblasts in breast cancer. Int J Biochem Cell Biol 2012; 44:2051-2059.

28 Allinen M, Beroukhim R, Cai L, et al. Molecular characterization of the tumor microenvironment in breast cancer. Cancer Cell 2004; 6:17-32.

29 Qiu W, Hu M, Sridhar A, et al. No evidence of clonal somatic genetic alterations in cancer-associated fibroblasts from human breast and ovarian carcinomas. Nat Genet 2008; 40:650655.

$30 \mathrm{Hu}$ M, Yao J, Cai L, et al. Distinct epigenetic changes in the stromal cells of breast cancer. Nat Genet 2005; 37:899-905.

31 Kalluri R, Zeisberg M. Fibroblasts in cancer. Nat Rev Cancer 2006; 6:392-401.

32 Xiong Y, Fang JH, Yun JP, et al. Effects of microRNA-29 on apoptosis, tumorigenicity, and prognosis of hepatocellular carcinoma. Hepatology 2010; 51:836-845.

33 Li Q, Zhang D, Wang Y, et al. MiR-21/Smad 7 signaling determines TGF- $\beta 1$-induced CAF formation. Sci Rep 2013; 3:2038.

34 van Rooij E, Sutherland LB, Thatcher JE, et al. Dysregulation of microRNAs after myocardial infarction reveals a role of miR-29 in cardiac fibrosis. Proc Natl Acad Sci USA 2008; 
105:13027-13032.

35 Rehmsmeier M, Steffen P, Hochsmann M, Giegerich R. Fast and effective prediction of microRNA/target duplexes. RNA 2004; 10:1507-1517.

36 Kalluri R, Weinberg RA. The basics of epithelial-mesenchymal transition. J Clin Invest 2009; 119:1420-1428.

37 Cirri P, Chiarugi P. Cancer associated fibroblasts: the dark side of the coin. Am J Cancer Res 2011; 1: 482-497.

38 Korkaya H, Liu S, Wicha MS. Regulation of cancer stem cells by cytokine networks: attacking cancer's inflammatory roots. Clin Cancer Res 2011; 17:6125-6129.

39 Nishikawa S, Konno M, Hamabe A, et al. Aldehyde dehydrogenase high gastric cancer stem cells are resistant to chemotherapy. Int J Oncol 2013; 42:1437-1442.

40 Fukuda K, Saikawa Y, Ohashi M, et al. Tumor initiating potential of side population cells in human gastric cancer. Int $J$ Oncol 2009; 34:1201-1207.

41 Lopez-Serra P, Esteller M. DNA methylation-associated silencing of tumor-suppressor microRNAs in cancer. Oncogene 2012; 31:1609-1622.

42 Creyghton MP, Cheng AW, Welstead GG, et al. Histone H3K27ac separates active from poised enhancers and predicts developmental state. Proc Natl Acad Sci USA 2010; 107:2193121936.

43 Xia D, Wang D, Kim SH, Katoh H, DuBois RN. Prostaglandin $\mathrm{E}_{2}$ promotes intestinal tumor growth via DNA methylation. Nat Med 2012; 18:224-226.

44 Raychaudhuri N, Douglas RS, Smith TJ. PGE2 induces IL-6 in orbital fibroblasts through EP2 receptors and increased gene promoter activity: implications to thyroid-associated ophthalmopathy. PLoS One 2010; 5:e15296.

45 Atherton JC. The pathogenesis of Helicobacter pylori-induced gastro-duodenal diseases. Ann Rev Pathol 2006; 1:63-96.

46 Akhtar M, Cheng Y, Magno RM, et al. Promoter methylation regulates Helicobacter pylori-stimulated cyclooxygenase-2 expression in gastric epithelial cells. Cancer Res 2001; 61:2399-2403.

47 McCarthy CJ, Crofford LJ, Greenson J, Scheiman JM. Cyclooxygenase-2 expression in gastric antral mucosa before and after eradication of Helicobacter pylori infection. Am J Gastroenterol 1999; 94:1218-1223.

48 Maekita T, Nakazawa K, Mihara M, et al. High levels of aberrant DNA methylation in Helicobacter pylori-infected gastric mucosae and its possible association with gastric cancer risk. Clin Cancer Res 2006; 12:989-995.

49 Ando T, Yoshida T, Enomoto S, et al. DNA methylation of microRNA genes in gastric mucosae of gastric cancer patients: its possible involvement in the formation of epigenetic field defect. Int J Cancer 2009; 124:2367-2374.

50 Zabaleta J. MicroRNA: a bridge from H. pylori infection to gastritis and gastric cancer development. Front Genet 2012; 3:294.

51 Liu H, Brannon AR, Reddy AR, et al. Identifying mRNA targets of microRNA dysregulated in cancer: with application to clear cell renal cell carcinoma. BMC Syst Biol 2010; 27:5167.

52 Pan SJ, Zhan SK, Pei BG, Sun QF, Bian LG, Sun BM. MicroRNA-149 inhibits proliferation and invasion of glioma cells via blockade of AKT1 signaling. Int J Immunopathol Pharmacol 2012; 25:871-881.
53 Luo Z, Zhang L, Li Z, et al. An in silico analysis of dynamic changes in microRNA expression profiles in stepwise development of nasopharyngeal carcinoma. BMC Med Genomics 2012; 19:3-14.

54 Jin L, Hu WL, Jiang CC, et al. MicroRNA-149*, a p53-responsive microRNA, functions as an oncogenic regulator in human melanoma. Proc Natl Acad Sci USA 2011; 108:1584015845.

55 Vrba L, Muñoz-Rodríguez JL, Stampfer MR, Futscher BW. miRNA gene promoters are frequent targets of aberrant DNA methylation in human breast cancer. PLoS One 2013; 8:e54398.

56 Grivennikov S, Karin E, Terzic J, et al. IL-6 and Stat3 are required for survival of intestinal epithelial cells and development of colitis-associated cancer. Cancer Cell 2009; 15:103113.

57 Bollrath J, Phesse TJ, von Burstin VA, et al. gp130-mediated Stat3 activation in enterocytes regulates cell survival and cell-cycle progression during colitis-associated tumorigenesis. Cancer Cell 2009; 15:91-102.

58 Guan J, Zhang H, Wen Z, et al. Retinoic acid inhibits pancreatic cancer cell migration and EMT through the downregulation of IL-6 in cancer associated fibroblast cells. Cancer Lett 2014; 345:132-139.

59 Santini P, Politi L, Vedova PD, Scandurra R, Scotto d'Abusco A. The inflammatory circuitry of miR-149 as a pathological mechanism in osteoarthritis. Rheumatol Int 2014; 34:711-716.

$60 \mathrm{Hu} \mathrm{S}$, Zhu W, Zhang LF, Pei M, Liu MF. MicroRNA-155 broadly orchestrates inflammation-induced changes of microRNA expression in breast cancer. Cell Res 2014; 24:254257.

61 Suzuki H, Maruyama R, Yamamoto E, Kai M. Epigenetic alteration and microRNA dysregulation in cancer. Front Genet 2013; 4:258.

62 Wang F, Ma YL, Zhang P, et al. SP1 mediates the link between methylation of the tumour suppressor miR-149 and outcome in colorectal cancer. J Pathol 2013; 229:12-24.

63 Wu X, Chen X, Zhou Q, et al. Hepatocyte growth factor activates tumor stromal fibroblasts to promote tumorigenesis in gastric cancer. Cancer Lett 2013; 335:128-135.

64 Lee A, O'Rourke J, De Ungria MC, Robertson B, Daskalopoulos G, Dixon MF. A standardized mouse model of Helicobacter pylori infection: introducing the Sydney strain. Gastroenterology 1997; 112:1386-1397.

65 Shigematsu Y, Niwa T, Rehnberg E, et al. Interleukin-1 $\beta$ induced by Helicobacter pylori infection enhances mouse gastric carcinogenesis. Cancer Lett 2013; 340:141-147.

66 Shimizu N, Kaminishi M, Tatematsu M, et al. Helicobacter pylori promotes development of pepsinogen-altered pyloric glands, a preneoplastic lesion of glandular stomach of BALB/ c mice pretreated with N-methyl-N-nitrosourea. Cancer Lett 1998; 123:63-69.

(Supplementary information is linked to the online version of the paper on the Cell Research website.)

(c) (1) (3) $\odot$ This work is licensed under the Creative Commons Attribution-NonCommercial-No Derivative Works 3.0 Unported License. To view a copy of this license, visit http:// creativecommons.org/licenses/by-nc-nd/3.0 